\title{
Rogers dilogarithms of higher degree and generalized cluster algebras
}

\author{
By Tomoki NAKANISHI
}

(Received Aug. 2, 2016)

(Revised Mar. 1, 2017)

\begin{abstract}
In connection with generalized cluster algebras we introduce a certain generalization of the celebrated Rogers dilogarithm, which we call the Rogers dilogarithms of higher degree. We show that there is an identity of these generalized Rogers dilogarithms associated with any period of seeds of a generalized cluster algebra.
\end{abstract}

\section{Introduction.}

It is widely known that the (Euler) dilogarithm

$$
\operatorname{Li}_{2}(x)=\sum_{n=1}^{\infty} \frac{x^{n}}{n^{2}}=-\int_{0}^{x} \frac{\log (1-y)}{y} d y
$$

appears and plays important roles in several branches of mathematics (e.g., [Lew81], [Kir95], [Zag07]). The function is remarkable in the sense that it satisfies a variety of functional equations, which are generally called dilogarithm identities.

The quantum dilogarithm [FV93], [FK94]

$$
\boldsymbol{\Psi}_{q}(x)=\prod_{k=0}^{\infty}\left(1+q^{2 k+1} x\right)^{-1},
$$

is regarded as a quantum analogue of the dilogarithm, and it is as important as the classical (i.e., nonquantum) one (1.1). It is related to its classical counterpart in the asymptotic limit as follows:

$$
\mathbf{\Psi}_{q}(x) \sim \exp \left(-\frac{\mathrm{Li}_{2}(-x)}{\log q^{2}}\right), \quad q \rightarrow 1^{-} .
$$

The classical and quantum dilogarithms are intimately related to the cluster algebras [FZ02], [FZ07] and quantum cluster algebras [FG09a], [FG09b], respectively. This connection was discovered via the quantization of the moduli space of Riemann surfaces [FC99], [FG09a], [FG09b]. Then, it was also spotlighted through the DonaldsonThomas theory [KS08], [Nag11], [Kel11], and through the $Y$-systems in conformal

2010 Mathematics Subject Classification. Primary 13F60; Secondary 33E20.

Key Words and Phrases. dilogarithm, quantum dilogarithm, cluster algebra.

This work was partially supported by JSPS KAKENHI Grant Number 16H03922. 
field theory [Cha05], [Nak11a], [Nak11b]. In particular, we reached to the following very general theorem.

THEOREM 1.1. (I). There is a dilogarithm identity associated with any period of seeds of a cluster algebra [Nak11b].

(II). There is a quantum dilogarithm identity associated with any period of seeds of a quantum cluster algebra [Kel11], [KN11].

A heuristic (but not rigorous) derivation of Statements (I) from Statement (II) was also given in [KN11].

Recently Chekhov and Shapiro introduced generalized cluster algebras [CS14]. As the name suggests it is a generalization of cluster algebras. In fact, it is a very natural generalization so that all nice properties of cluster algebras are shown or expected to hold [Rup13], [Nak15b]. Naturally one can further define the quantum generalized cluster algebras as well [Nak15a]. For ordinary quantum cluster algebras, the quantum dilogarithm (1.2) plays a key role to control their mutations [FG09a], [FG09b]. Likewise, for generalized quantum cluster algebras, a generalization of the quantum dilogarithm, called the quantum dilogarithms of higher degree play the same role, and they look as follows: (This notation is used only here.)

$$
\Psi_{q}^{P}(x)=\prod_{k=0}^{\infty} P\left(q^{2 k+1} x\right)^{-1},
$$

where $P(x)$ is an arbitrary monic polynomial in $x$ with unit constant; furthermore, here we assume that all coefficients of $P(x)$ are nonnegative real numbers.

In the simplest case $\operatorname{deg} P(x)=1$, we have $P(x)=1+x$ and it reduces to the ordinary one (1.2). (In [Nak15a] $P(x)$ is assumed to be reciprocal, but this assumption can be removed with slight change of mutations therein. See [NR16].) Then, it is rather straightforward to generalize Statement (II) in Theorem 1.1 in the following way:

TheOREm 1.2. (II'). There is a quantum dilogarithm identity of higher degree associated with any period of seeds of a quantum generalized cluster algebra [Nak15a].

Under this circumstance it is just natural to expect the following generalization of Statement (I), which is also the classical counterpart of Statement (II'):

(I'). There is a dilogarithm identity of higher degree associated with any period of seeds of a generalized cluster algebra.

In fact, a classical counterpart of the function (1.4) was already introduced in [Nak15a], and it looks as follows: (This notation is used only here.)

$$
\operatorname{Li}_{2}^{P}(x)=-\int_{0}^{-x} \frac{\log P(y)}{y} d y .
$$

Two functions in (1.4) and (1.5) are related in the same way as (1.3): 


$$
\mathbf{\Psi}_{q}^{P}(x) \sim \exp \left(-\frac{\operatorname{Li}_{2}^{P}(-x)}{\log q^{2}}\right), \quad q \rightarrow 1^{-} .
$$

Now let us explain the obstacle to establish Statement (I') we have been confronted with so far. In the ordinary case the derivation of Statement (I) in [Nak11b] is not as straightforward as its quantum counterpart (II). The reason is that the dilogarithm naturally concerning with the identities in Statement (I) is not exactly the Euler dilogarithm (1.1); rather, it is the Rogers dilogarithm defined by

$$
L(x)=-\frac{1}{2} \int_{0}^{x}\left\{\frac{\log (1-y)}{y}+\frac{\log y}{1-y}\right\} d y,
$$

where two dilogarithms are related by

$$
L(x)=\operatorname{Li}_{2}(x)+\frac{1}{2} \log x \cdot \log (1-x) .
$$

Then, the main issue is to set the proper definition of the Rogers dilogarithms of higher degree so as to match the desired Statement (I'), which is not a priori clear.

In this paper we resolve this problem, and we give a proper definition of the Rogers dilogarithms of higher degree. The key idea is to generalize the Rogers dilogarithm, not through the familiar definition (1.7), but through a less familiar expression,

$$
L\left(\frac{x}{1+x}\right)=\frac{1}{2} \int_{0}^{x}\left\{\frac{\log (1+y)}{y}-\frac{\log y}{1+y}\right\} d y, \quad(0 \leq x) .
$$

Then, for the same polynomial $P(x)$ in (1.5), we define the corresponding Rogers dilogarithm of higher degree as follows: (This notation is used only here.)

$$
L^{P}\left(\frac{x^{\operatorname{deg} P}}{P(x)}\right)=\frac{1}{2} \int_{0}^{x}\left\{\frac{\log P(y)}{y}-\frac{\log y}{P(y)} P^{\prime}(y)\right\} d y, \quad(0 \leq x) .
$$

Once this part is cleared, it is rather straightforward to follow the argument of the proof of Statement (I), and we prove Statement (I'), which is our main result (Theorems 4.7 and 4.8). To complete the picture, we also give a heuristic derivation of Statement (I') from Statement (II') following [KN11].

The function (1.10) may formally reduce to (the analytic continuation of) the ordinary one (1.9) if we factorize the polynomial $P(x)$ into polynomials of degree one with complex coefficients. However, we need to correctly choose the path of the analytic continuation of (1.9) very carefully, and that makes the things very complicated. Therefore, it is natural to keep the function (1.10) as a single package.

The organization of the paper is as follows. In Section 2 the connection between the Rogers dilogarithm and cluster algebras is reviewed. In Section 3 we introduce the Rogers dilogarithms of higher degree and prove the constancy condition. In Section 4 we show that there is a dilogarithm identity of higher degree associated with any period of a generalized cluster algebra. In Section 5 we give a proof of Theorem 4.4, which is the key theorem for our main result. In Section 6 some explicit examples are given. In Appendix, as independent reading, we give a heuristic (but not rigorous) derivation of 
our dilogarithm identity of higher degree from the quantum one.

ACKNowledgements. The author would like to thank the referee for useful comments and suggestions.

\section{Rogers dilogarithm and cluster algebras.}

In this section we quickly recall and summarize some known properties of the Rogers dilogarithm and its relation to cluster algebras, which we will generalize in this paper. The whole section may serve as a useful guide to show where we are heading. Here we do not provide any proof, but the main theorems will be reproved in a more general setting in the later sections.

\subsection{Rogers dilogarithm.}

The Euler dilogarithm $\mathrm{Li}_{2}(x)$ was originally defined by the following power series with convergence radius 1 (see [Lew81], [Kir95], [Zag07] for backgrounds):

$$
\mathrm{Li}_{2}(x)=\sum_{n=1}^{\infty} \frac{x^{n}}{n^{2}}
$$

We have

$$
\operatorname{Li}_{2}(0)=0, \quad \operatorname{Li}_{2}(1)=\zeta(2)=\frac{\pi^{2}}{6}
$$

where $\zeta(s)$ is the Riemann zeta function. We also have the integral expression

$$
\operatorname{Li}_{2}(x)=-\int_{0}^{x} \frac{\log (1-y)}{y} d y=-\int_{0}^{-x} \frac{\log (1+y)}{y} d y, \quad(x \leq 1) .
$$

The integrals in (2.3) can be extended on the whole complex plane $\mathbb{C}$. However, there is a branch point at $x=1$, and the function $\operatorname{Li}_{2}(x)$ becomes multivalued on $\mathbb{C}$. Here we restrict our attention only on the region $x \leq 1$ so that there is no ambiguity of multivaluedness.

The Rogers dilogarithm $L(x)$ is defined by

$$
\begin{aligned}
L(x) & =-\frac{1}{2} \int_{0}^{x}\left\{\frac{\log (1-y)}{y}+\frac{\log y}{1-y}\right\} d y, \quad(0 \leq x \leq 1), \\
& =\operatorname{Li}_{2}(x)+\frac{1}{2} \log x \cdot \log (1-x) .
\end{aligned}
$$

Again, we restrict our attention only on the region $0 \leq x \leq 1$, and there is no ambiguity of multivaluedness.

By (2.2), we have

$$
L(0)=0, \quad L(1)=\frac{\pi^{2}}{6}
$$

We also have 


$$
L(x)+L(1-x)=L(1), \quad(0 \leq x \leq 1) .
$$

The following equalities hold:

$$
\begin{aligned}
L\left(\frac{x}{1+x}\right) & =\frac{1}{2} \int_{0}^{x}\left\{\frac{\log (1+y)}{y}-\frac{\log y}{1+y}\right\} d y, \quad(0 \leq x) \\
& =-\operatorname{Li}_{2}(-x)-\frac{1}{2} \log x \cdot \log (1+x)
\end{aligned}
$$

These equalities are less well-known than (2.4) and (2.5), but they are crucial for our purpose. They are easily proven by taking the derivative. Since the function $x /(1+x)$ is monotonic on $\mathbb{R}_{\geq 0}$ and yields a bijection from $\mathbb{R}_{\geq 0}$ to $[0,1)$, one may view $(2.8)$ and $(2.9)$ as an alternative definition of $L(x)$ on $[0,1]$, where $L(1)$ is defined by the limit $x \rightarrow \infty$ in $(2.8)$.

In view of the form (2.8), it is useful to rephrase the equality (2.7) as

$$
L\left(\frac{x}{1+x}\right)+L\left(\frac{1}{1+x}\right)=L(1), \quad(0 \leq x) .
$$

Note that there is a duality of the variables in (2.10):

$$
\frac{1}{1+x}=\left.\frac{x}{1+x}\right|_{x=x^{-1}}
$$

\subsection{Constancy condition.}

Let $G$ be any multiplicative abelian group. Let $G \otimes G$ be its tensor product over $\mathbb{Z}$, that is, the additive abelian group with generators $f \otimes g(f, g \in G)$ and relations

$$
(f g) \otimes h=f \otimes h+g \otimes h, \quad f \otimes(g h)=f \otimes g+f \otimes h .
$$

Note that $1 \otimes h=h \otimes 1=0$. Let $S^{2} G$ be the symmetric subgroup of $G \otimes G$, namely, the subgroup generated by all $f \otimes g+g \otimes f(f, g \in G)$. We define the wedge product of $G$ as $\bigwedge^{2} G=G \otimes G / S^{2} G$.

Let $\mathcal{C}=\mathcal{C}\left([0,1], \mathbb{R}_{+}\right)$be the set of all positive-real-valued and differentiable functions on the interval $[0,1]$ in $\mathbb{R}$. Regarding it as a multiplicative abelian group, we have the wedge product $\bigwedge^{2} \mathcal{C}$.

The following theorem is the starting point of deducing identities satisfied by the Rogers dilogarithm.

TheOREM 2.1 ([FS95, Proposition 1]). Let $f_{1}, \ldots, f_{m} \in \mathcal{C}$ be differentiable functions on the interval $[0,1]$ which especially takes values in the interval $(0,1)$. Suppose that they satisfy the following relation in $\bigwedge^{2} \mathcal{C}$ : (Constancy condition)

$$
\sum_{t=1}^{m} f_{t} \wedge\left(1-f_{t}\right)=0 .
$$

Then, the sum of the Rogers dilogarithm 


$$
\sum_{t=1}^{m} L\left(f_{t}(u)\right)
$$

is constant as a function of $u \in[0,1]$.

In view of the form (2.8), it is useful to rephrase Theorem 2.1 as follows:

Theorem 2.2 (cf. [Cha05, Equation (2.3)]). Let $y_{1}, \ldots, y_{m} \in \mathcal{C}$. Suppose that they satisfy the following the relation in $\wedge^{2} \mathcal{C}$ : (Constancy condition)

$$
\sum_{t=1}^{m} y_{t} \wedge\left(1+y_{t}\right)=0 .
$$

Then, the sum of the Rogers dilogarithm

$$
\sum_{t=1}^{m} L\left(\frac{y_{t}(u)}{1+y_{t}(u)}\right)
$$

is constant as a function of $u \in[0,1]$.

\subsection{Seed mutations.}

To make use of wonderful Theorem 2.2, it is essential to find a family of functions $y_{1}, \ldots, y_{m} \in \mathcal{C}$ which satisfy the constancy condition (2.15). This is where cluster algebras take part. See [FZ07] for a general reference on cluster algebras.

First we recall the notion of a semifield, following [FZ07].

Definition 2.3. A multiplicative abelian group $\mathbb{P}$ is called a semifield if it is endowed with a binary operation $\oplus$ which is commutative, associative, and distributative, i.e., $a(b \oplus c)=a b \oplus a c$. The operation $\oplus$ is called the addition.

Here we mainly use the following examples.

Example 2.4. (1) The set $\mathbb{R}_{+}$of all positive real numbers is a semifield, where the product and the addition are given by the usual ones.

(2) Let $y=\left(y_{i}\right)_{i=1}^{n}$ be an $n$-tuple of formal commutative variables. We say that a rational function $f(y)$ in $y$ with coefficients in $\mathbb{Q}$ has a subtraction-free expression if it is represented as $f(y)=P(y) / Q(y)$ such that $P(y)$ and $Q(y)$ are nonzero polynomials in $y$ with nonnegative integer coefficients. Let $\mathbb{Q}_{+}(y)$ be the set of all rational functions in $y$ having subtraction-free expressions. Then, $\mathbb{Q}_{+}(y)$ is a semifield, where the product and the addition are given by the usual ones for rational functions. We call it the universal semifield of $y$.

(3) Let $y=\left(y_{i}\right)_{i=1}^{n}$ be an $n$-tuple of formal commutative variables. Let

$$
\operatorname{Trop}(y)=\left\{\prod_{i=1}^{n} y_{i}^{a_{i}} \mid a_{i} \in \mathbb{Z}\right\} .
$$

Then, $\operatorname{Trop}(y)$ is a semifield, where the product is given by the usual one for Laurent 
monomials, while the addition is given by the following tropical sum:

$$
\prod_{i=1}^{n} y_{i}^{a_{i}} \oplus \prod_{i=1}^{n} y_{i}^{b_{i}}=\prod_{i=1}^{n} y_{i}^{\min \left(a_{i}, b_{i}\right)} .
$$

We call it the tropical semifield of $y$.

Let $\mathbb{P}$ be any semifield. By regarding it as an abelian multiplicative group, let $\mathbb{Z}[\mathbb{P}]$ be its group ring. It is known that $\mathbb{Z}[\mathbb{P}]$ is a domain $[\mathbf{F Z 0 2}]$, i.e., there is no divisor. Thus, the field of the fractions of $\mathbb{Z}[\mathbb{P}]$ is well-defined, and it is denoted by $\mathbb{Q}(\mathbb{P})$ here.

We say that an (integer) square matrix $B$ is skew-symmetrizable if there is a diagonal matrix $R=\operatorname{diag}\left(r_{1}, \ldots, r_{n}\right)$ of the same size with positive (integer) diagonal entries $r_{1}, \ldots, r_{n}$ such that $R B$ is skew-symmetric, i.e., $(R B)^{T}=-R B$. Also we call such $R$ a skew-symmetrizer of $B$. Note that if $R B$ is skew-symmetric, then $B R^{-1}$ is also skew-symmetric.

Now let us give two most important notions in cluster algebras, namely, a seed and its mutation. (We do not give the definition of a cluster algebra itself, because it is not essential in this paper.)

Definition 2.5. Let us fix a positive integer $n \in \mathbb{Z}_{+}$and a semifield $\mathbb{P}$, which are called the rank and the coefficient semifield (of a cluster algebra under consideration). Let $w=\left(w_{i}\right)_{i=1}^{n}$ be an $n$-tuple of formal commutative variables, and let $\mathcal{F}=(\mathbb{Q}(\mathbb{P}))(w)$ be the rational function field of $w$ with coefficients in $\mathbb{Q}(\mathbb{P})$. We call $\mathcal{F}$ the ambient field.

(1). Let $(B, x, y)$ be a triplet such that

- $B=\left(b_{i j}\right)_{i, j=1}^{n}$ is a skew-symmetrizable integer matrix of size $n$,

- $x=\left(x_{i}\right)_{i=1}^{n}$ is an $n$-tuple of algebraically independent elements of $\mathcal{F}$,

- $y=\left(y_{i}\right)_{i=1}^{n}$ is an $n$-tuple of elements of $\mathbb{P}$.

We call such $(B, x, y)$ a seed, and call $B, x$, and $y$ the exchange matrix, the $x$-variables, and the $y$-variables of a seed $(B, x, y)$, respectively. Also, we set

$$
\hat{y}_{i}=y_{i} \prod_{j=1}^{n} x_{j}^{b_{j i}} \in \mathcal{F}, \quad i=1, \ldots, n,
$$

and call them the $\hat{y}$-variables of a seed $(B, x, y)$.

$(2)$. For any seed $(B, x, y)$ and any $k=1, \ldots, n$, we define a new seed $\left(B^{\prime}, x^{\prime}, y^{\prime}\right)=$ $\mu_{k}(B, x, y)$, called the mutation of $(B, x, y)$ at $k$, as follows:

$$
\begin{aligned}
& b_{i j}^{\prime}= \begin{cases}-b_{i j} & i=k \text { or } j=k \\
b_{i j}+\left[-b_{i k}\right]_{+} b_{k j}+b_{i k}\left[b_{k j}\right]_{+} & i, j \neq k,\end{cases} \\
& x_{i}^{\prime}= \begin{cases}x_{k}^{-1}\left(\prod_{j=1}^{n} x_{j}^{\left[-b_{j k}\right]_{+}}\right) \frac{1+\hat{y}_{k}}{1 \oplus y_{k}} & i=k \\
x_{i} & i \neq k,\end{cases}
\end{aligned}
$$




$$
y_{i}^{\prime}= \begin{cases}y_{k}^{-1} & i=k \\ y_{i} y_{k}^{\left[b_{k i}\right]_{+}}\left(1 \oplus y_{k}\right)^{-b_{k i}} & i \neq k .\end{cases}
$$

Here and elsewhere, for any integer $a$, we define

$$
[a]_{+}=\max (a, 0) .
$$

The following facts can be easily checked:

(1). The mutation $\mu_{k}$ is an involution, i.e., $\mu_{k}\left(\mu_{k}(B, x, y)\right)=(B, x, y)$.

(2). If $R$ is a skew-symmetrizer of $B$, then $R$ is also a skew-symmetrizer of $B^{\prime}$.

(3). The $\hat{y}$-variables transform in $\mathcal{F}$ as the $y$-variables; namely,

$$
\hat{y}_{i}^{\prime}= \begin{cases}\hat{y}_{k}^{-1} & i=k \\ \hat{y}_{i} \hat{y}_{k}^{\left[b_{k i}\right]_{+}}\left(1+\hat{y}_{k}\right)^{-b_{k i}} & i \neq k .\end{cases}
$$

\subsection{Dilogarithm identities.}

In this section we specialize the coefficient semifield $\mathbb{P}$ in Definition 2.5 as $\mathbb{P}=\mathbb{Q}_{+}(y)$ in Example 2.4 (2) with generators $y=\left(y_{i}\right)_{i=1}^{n}$. Let us choose a seed $(B, x, y)$, where $B$ and $x$ are arbitrary, but we especially choose $y$ to be the generators of $\mathbb{Q}_{+}(y)$. Let us call $(B, x, y)$ the initial seed, and consider a sequence of mutations starting from it:

$$
\begin{aligned}
(B, x, y)=(B[1], x[1], y[1]) & \stackrel{\mu_{k_{1}}}{\rightarrow}(B[2], x[2], y[2]) \stackrel{\mu_{k_{2}}}{\rightarrow} \\
& \cdots \stackrel{\mu_{k_{m}}}{\rightarrow}(B[m+1], x[m+1], y[m+1]) .
\end{aligned}
$$

Let $R=\operatorname{diag}\left(r_{1}, \ldots, r_{n}\right)$ be a common symmetrizer of $B[1], \ldots, B[m+1]$, and let $r$ be the least common multiple of $r_{1}, \ldots, r_{n}$. We set $\tilde{r}_{i}=r / r_{i} \in \mathbb{Z}_{+}$.

Definition 2.6. We say that the sequence (2.25) is $\sigma$-periodic for a permutation $\sigma$ of $\{1, \ldots, n\}$ if

$$
b_{\sigma(i) \sigma(j)}[m+1]=b_{i j}, \quad x_{\sigma(i)}[m+1]=x_{i}, \quad y_{\sigma(i)}[m+1]=y_{i}, \quad(i, j=1, \ldots, n) .
$$

The following fact connects cluster algebras and dilogarithm identities.

Theorem 2.7 ([Nak11b, Proposition 6.3 and Section 6.5]). Suppose that the sequence (2.25) is $\sigma$-periodic for some permutation $\sigma$. Then, it satisfies the following "constancy condition" in the wedge product $\bigwedge^{2} \mathbb{Q}_{+}(y)$, where we regard $\mathbb{Q}_{+}(y)$ as a multiplicative abelian group:

$$
\sum_{t=1}^{m} \tilde{r}_{k_{t}}\left(y_{k_{t}}[t] \wedge\left(1+y_{k_{t}}[t]\right)\right)=0 .
$$

Combining Theorems 2.2 and 2.7, we obtain the first half of the dilogarithm identity associated with any period of seeds. 
TheOREM 2.8 ([Nak11b, Theorems 6.4 and 6.8]). Suppose that the sequence (2.25) is $\sigma$-periodic for some permutation $\sigma$. Let

$$
\varphi: \mathbb{Q}_{+}(y) \rightarrow \mathbb{R}_{+}
$$

be any semifield homomorphism. Then, the following sum does not depend on the choice of $\varphi$ :

$$
\sum_{t=1}^{m} \tilde{r}_{k_{t}} L\left(\varphi\left(\frac{y_{k_{t}}[t]}{1+y_{k_{t}}[t]}\right)\right) \text {. }
$$

REMARK 2.9. Theorem 6.8 in [Nak11b] was proved under the assumption of the sign-coherence property of the $c$-vectors, which is now proved by [GHKK18]. See Theorem 2.10 below.

The second half of the dilogarithm identity is about the constant value of (2.29). To describe it, we introduce the semifield homomorphism (tropicalization map)

$$
\begin{aligned}
\pi: \mathbb{Q}_{+}(y) & \rightarrow \operatorname{Trop}(y) \\
y_{i} & \mapsto y_{i} .
\end{aligned}
$$

We then apply the map $\pi$ to each $y$-variable $y_{i}[t]$ in (2.25), and express it as

$$
\pi\left(y_{i}[t]\right)=\prod_{j=1}^{n} y_{j}^{c_{j i}[t]}
$$

Thus, we have a family of square matrices $C[t]=\left(c_{i j}[t]\right)_{i, j=1}^{n}$ for $t=1, \ldots, m+1$, which are called the $C$-matrices for the sequence (2.25). Alternatively, they can be directly defined through the following system of recursion relations [FZ07]:

(initial condition)

$$
c_{i j}[1]=\delta_{i j}
$$

(recursion relation)

$$
c_{i j}[t+1]= \begin{cases}-c_{i k_{t}}[t] & j=k_{t} \\ c_{i j}[t]+\left[-c_{i k_{t}}[t]\right]_{+} b_{k_{t} j}[t]+c_{i k_{t}}[t]\left[b_{k_{t} j}[t]\right]_{+} & j \neq k_{t} .\end{cases}
$$

The $i$ th column vector $c_{i}[t]=\left(c_{j i}[t]\right)_{j=1}^{n}$ of the matrix $C[t]$ is called the $c$-vector of $y_{i}[t]$. By the definition of (2.31), it is the "exponent vector" of the tropical $y$-variable $\pi\left(y_{i}[t]\right)$.

The following property is fundamental in the theory of cluster algebras, and it is originally conjectured by [FZ07] and proved in full generality recently:

THEOREM 2.10 ((Sign coherence) [GHKK18, Corollary 5.5]). Each c-vector $c_{i}[t]$ is a nonzero vector, and all its components are either nonnegative or nonpositive.

Accordingly, we set the tropical sign $\varepsilon\left(y_{i}[t]\right) \in\{ \pm 1\}$ of $y_{i}[t]$ as 1 (resp. - 1 ) if all 
components of $c_{i}[t]$ is nonnegative (resp. nonpositive). For simplicity, let us write

$$
\varepsilon_{t}=\varepsilon\left(y_{k_{t}}[t]\right) .
$$

Now, continuing from Theorem 2.8, we can state the second half of the dilogarithm identity.

TheOREM 2.11 ([Nak11b, Theorems 6.4 and 6.8]). Under the assumption of Theorem 2.8, we have the following equality for any choice of $\varphi$ in (2.28):

$$
\sum_{t=1}^{m} \tilde{r}_{k_{t}} L\left(\varphi\left(\frac{y_{k_{t}}[t]}{1+y_{k_{t}}[t]}\right)\right)=\sum_{t=1}^{m} \frac{1-\varepsilon_{t}}{2} \tilde{r}_{k_{t}} L(1) .
$$

Using (2.10), we also have an alternative form of the identity (2.35), which is constant-term free.

TheOREM 2.12 ([KN11, Theorem 2.9]). The identity (2.35) is equivalent to the following one:

$$
\sum_{t=1}^{m} \varepsilon_{t} \tilde{r}_{k_{t}} L\left(\varphi\left(\frac{\left(y_{k_{t}}[t]\right)^{\varepsilon_{t}}}{1+\left(y_{k_{t}}[t]\right)^{\varepsilon_{t}}}\right)\right)=0 .
$$

We are going to generalize Theorems 2.11 and 2.12 based on generalized cluster algebras.

\section{Rogers dilogarithms of higher degree.}

In this section we introduce the Rogers dilogarithms of higher degree. Then, we prove the constancy condition which is parallel to Theorem 2.2.

\subsection{Rogers dilogarithms of higher degree.}

Let $d$ be any positive integer, and let $z=\left(z_{s}\right)_{s=0}^{d}$ such that $z_{0}=z_{d}=1$ and $z_{1}, \ldots, z_{d-1} \in \mathbb{R}_{\geq 0}$ are arbitrary. Let $P_{d, z}(x)$ be the polynomial in a single variable $x$ defined by

$$
P_{d, z}(x)=\sum_{s=0}^{d} z_{s} x^{s}
$$

Below we assume the following generic condition for $z$ :

The polynomial $P_{d, z}(x)$ has no root on $\mathbb{R}$ except for $x=-1$.

In $\left[\mathbf{N a k 1 5 b}\right.$ the (Euler) dilogarithm $\mathrm{Li}_{2 ; d, z}(x)$ of degree $d$ with coefficients $z$ is defined as follows (cf. (2.3)):

$$
\operatorname{Li}_{2 ; d, z}(x)=-\int_{0}^{-x} \frac{\log P_{d, z}(y)}{y} d y, \quad(x \leq 1) .
$$


By the condition (3.2), there is no ambiguity of multivaluedness in the region $x \leq 1$.

In view of (2.8) and (2.9) we define the Rogers dilogarithm $L_{d, z}(x)$ of degree $d$ with coefficients $z$ as follows:

$$
\begin{aligned}
L_{d, z}\left(\frac{x^{d}}{P_{d, z}(x)}\right) & =\frac{1}{2} \int_{0}^{x}\left\{\frac{\log P_{d, z}(y)}{y}-\frac{\log y}{P_{d, z}(y)} P_{d, z}^{\prime}(y)\right\} d y, \quad(0 \leq x), \\
& =-\operatorname{Li}_{2 ; d, z}(-x)-\frac{1}{2} \log x \cdot \log P_{d, z}(x),
\end{aligned}
$$

where $P_{d, z}^{\prime}(x)$ denotes the derivative of $P_{d, z}(x)$. Since the function $x^{d} / P_{d, z}(x)$ is monotonic on $\mathbb{R}_{\geq 0}$ and yields a bijection from $\mathbb{R}_{\geq 0}$ to $[0,1)$, (3.4) and (3.5) unambiguously determine the function $L_{d, z}(x)$ on $[0,1]$, where $L_{d, z}(1)$ is defined by the limit $x \rightarrow \infty$ in (3.4).

For the above $z=\left(z_{s}\right)_{s=0}^{d}$, we define its reverse $z^{*}$ as

$$
z^{*}=\left(z_{s}^{*}\right)_{s=0}^{d}, \quad z_{s}^{*}=z_{d-s}
$$

Clearly, it holds that $\left(z^{*}\right)^{*}=z$. Also, we have

$$
\begin{aligned}
P_{d, z^{*}}\left(x^{-1}\right) & =x^{-d} P_{d, z}(x), \\
\left.P_{d, z^{*}}^{\prime}(x)\right|_{x=x^{-1}} & =-x^{2-d} P_{d, z}^{\prime}(x)+d x^{1-d} P_{d, z}(x) .
\end{aligned}
$$

By (3.7), $a \neq 0$ is a root of $P_{d, z^{*}}(x)$ if and only if $a^{-1}$ is a root of $P_{d, z}(x)$. Thus, $z^{*}$ also satisfies the generic condition (3.2).

The following is the counterpart of the equalities (2.10) and (2.11):

Proposition 3.1. We have the equalities

$$
\begin{gathered}
L_{d, z}\left(\frac{x^{d}}{P_{d, z}(x)}\right)+L_{d, z^{*}}\left(\frac{1}{P_{d, z}(x)}\right)=L_{d, z}(1)=L_{d, z^{*}}(1), \quad(0 \leq x), \\
\frac{1}{P_{d, z}(x)}=\left.\frac{x^{d}}{P_{d, z^{*}}(x)}\right|_{x=x^{-1}} .
\end{gathered}
$$

Proof. The equality (3.10) is immediate from (3.7). Let us show (3.9). First, we show that the left-hand side of (3.9) does not depend on $x$. To do it, we apply (3.10) in the second term of the left-hand side of (3.9), then take the derivative with respect to $x$. Then, by (3.4), we obtain

$$
\begin{aligned}
& \frac{1}{2}\left(\frac{\log P_{d, z}(x)}{x}-\frac{\log x}{P_{d, z}(x)} P_{d, z}^{\prime}(x)\right) \\
& \quad-\frac{1}{2} x^{-2}\left(\frac{\log P_{d, z^{*}}\left(x^{-1}\right)}{x^{-1}}-\frac{\log x^{-1}}{P_{d, z^{*}}\left(x^{-1}\right)}\left(\left.P_{d, z^{*}}^{\prime}(x)\right|_{x=x^{-1}}\right)\right)
\end{aligned}
$$

Then, using (3.7) and (3.8), it is easy to check that (3.11) vanishes. Thus, the left-hand side of (3.9) is a constant $C$ with respect to $x$. Then, taking $x \rightarrow \infty$ in it, we have $C=L_{d, z}(1)$, while setting $x=0$, we have $C=L_{d, z^{*}}(1)$. 
Let us also introduce the function $\tilde{L}_{d, z}(x)$ by

$$
\tilde{L}_{d, z}(x):=L_{d, z}\left(\frac{x^{d}}{P_{d, z}(x)}\right), \quad(0 \leq x) .
$$

Then, by (3.10), the equality (3.9) is written as

$$
\tilde{L}_{d, z}(x)+\tilde{L}_{d, z^{*}}\left(x^{-1}\right)=\tilde{L}_{d, z}(\infty)=\tilde{L}_{d, z^{*}}(\infty), \quad(0 \leq x),
$$

where $\tilde{L}_{d, z}(\infty):=\lim _{x \rightarrow \infty} \tilde{L}_{d, z}(x)$. Though we are attached to the function $L_{d, z}(x)$, since it is more directly related to the classic Rogers dilogarithm $L(x)$, all results in this paper are more simply described with the function $\tilde{L}_{d, z}(x)$ as (3.13). So, from now on, we mainly use the function $\tilde{L}_{d, z}(x)$ instead of $L_{d, z}(x)$.

\subsection{Constancy condition.}

Recall that $\mathcal{C}=\mathcal{C}\left([0,1], \mathbb{R}_{+}\right)$is the set of all positive-real-valued and differentiable functions on the interval $[0,1]$ as defined in Section 2.2 .

Theorem 3.2 (cf. Theorem 2.2). Let $y_{1}, \ldots, y_{m} \in \mathcal{C}$, and, for $t=1, \ldots, m$, let $P_{d_{t}, z_{t}}(x)$ be a degree $d_{t}$ polynomial in $x$ whose coefficients $z_{t}$ satisfy the generic condition (3.2). Suppose that they satisfy the following relation in $\bigwedge^{2} \mathcal{C}$ : (Constancy condition)

$$
\sum_{t=1}^{m} y_{t} \wedge P_{d_{t}, z_{t}}\left(y_{t}\right)=0 .
$$

Then, the sum of the Rogers dilogarithms of higher degree

$$
\sum_{t=1}^{m} \tilde{L}_{d_{t}, z_{t}}\left(y_{t}(u)\right)
$$

is constant as a function of $u \in[0,1]$.

Proof. The proof essentially repeats the one for Theorem 2.1 due to [FS95], whose idea originates in [Blo78]. By (3.4), for each $t=1, \ldots, m$, we have

$$
\begin{aligned}
\frac{d}{d u} \tilde{L}_{d_{t}, z_{t}}\left(y_{t}(u)\right)=\frac{1}{2} & \left(\log P_{d_{t}, z_{t}}\left(y_{t}(u)\right) \cdot \frac{d}{d u} \log y_{t}(u)\right. \\
& \left.-\log y_{t}(u) \cdot \frac{d}{d u} \log P_{d_{t}, z_{t}}\left(y_{t}(u)\right)\right) .
\end{aligned}
$$

On the other hand, by the assumption of (3.14),

$$
\sum_{t=1}^{m} y_{t} \otimes P_{d_{t}, z_{t}}\left(y_{t}\right)=\sum_{i=1}^{k}\left(g_{i} \otimes h_{i}+h_{i} \otimes g_{i}\right)
$$

for some $k \geq 1$ and $g_{i}, h_{i} \in \mathcal{C}$. For any $u, v \in[0,1]$, we have an additive group homomorphism $\Psi_{u, v}: \mathcal{C} \otimes \mathcal{C} \rightarrow \mathbb{R}, f \otimes g \mapsto \log f(u) \cdot \log g(v)$. Applying it on (3.17), we have 


$$
\sum_{t=1}^{m} \log y_{t}(u) \cdot \log P_{d_{t}, z_{t}}\left(y_{t}(v)\right)=\sum_{i=1}^{k}\left(\log g_{i}(u) \cdot \log h_{i}(v)+\log h_{i}(u) \cdot \log g_{i}(v)\right) .
$$

Then, taking the derivative for $u$ and setting $v=u$, we have

$$
\begin{aligned}
& \sum_{t=1}^{m} \frac{d}{d u} \log y_{t}(u) \cdot \log P_{d_{t}, z_{t}}\left(y_{t}(u)\right) \\
& =\sum_{i=1}^{k}\left(\frac{d}{d u} \log g_{i}(u) \cdot \log h_{i}(u)+\frac{d}{d u} \log h_{i}(u) \cdot \log g_{i}(u)\right) .
\end{aligned}
$$

Similarly, taking the derivative for $v$ and setting $v=u$, we have

$$
\begin{aligned}
& \sum_{t=1}^{m} \log y_{t}(u) \cdot \frac{d}{d u} \log P_{d_{t}, z_{t}}\left(y_{t}(u)\right) \\
& =\sum_{i=1}^{k}\left(\log g_{i}(u) \cdot \frac{d}{d u} \log h_{i}(u)+\log h_{i}(u) \cdot \frac{d}{d u} \log g_{i}(u)\right) .
\end{aligned}
$$

Note that the right-hand sides of (3.19) and (3.20) are identical. Thus, by (3.16), (3.19), and (3.20), we obtain the equality

$$
\frac{d}{d u} \sum_{t=1}^{m} \tilde{L}_{d_{t}, z_{t}}\left(y_{t}(u)\right)=0
$$

\section{Identities associated with periods of generalized cluster algebras.}

In this section we show Statement (II') in Section 1; that is, there is a dilogarithm identity of higher degree associated with any period of seeds of a generalized cluster algebra.

\subsection{Seed mutations for generalized cluster algebras.}

Generalized cluster algebras were introduced by [CS14]. Here we present the definitions of a seed and its mutation for a generalized cluster algebra, following [Nak15b], [NR16]. (Again, we do not give the definition of a generalized cluster algebra itself, because it is not essential in this paper.)

Definition 4.1. Let us fix a positive integer $n$ and a semifield $\mathbb{P}$, which are called the rank and the coefficient semifield (of a generalized cluster algebra under consideration). In addition, we also fix an $n$-tuple $d=\left(d_{i}\right)_{i=1}^{n}$ of positive integers, which is called the mutation degree. For the simplest choice $d=(1, \ldots, 1)$, it reduces to the ordinary cluster algebra case. Let $w=\left(w_{i}\right)_{i=1}^{n}$ be an $n$-tuple of formal commutative variables and let $\mathcal{F}=(\mathbb{Q}(\mathbb{P}))(w)$ be the rational function field of $w$ with coefficients in $\mathbb{Q}(\mathbb{P})$, which is called the ambient field.

(1). Let $(B, x, y, z)$ be a quartet such that

- $B=\left(b_{i j}\right)_{i, j=1}^{n}$ is a skew-symmetrizable integer matrix of size $n$, 
- $x=\left(x_{i}\right)_{i=1}^{n}$ is an $n$-tuple of algebraically independent elements of $\mathcal{F}$,

- $y=\left(y_{i}\right)_{i=1}^{n}$ is an $n$-tuple of elements of $\mathbb{P}$.

- $z=\left(z_{i, s} \mid i=1, \ldots, n ; s=0, \ldots, d_{i}\right)$ is a collection of elements of $\mathbb{P}$ such that $z_{i, 0}=z_{i, d_{i}}=1$ for any $i=1, \ldots, n$.

Here we call such $(B, x, y, z)$ a seed, and call $B, x, y$, and $z$ the exchange matrix, the $x$-variables, the $y$-variables, and $z$-variables of a seed $(B, x, y, z)$, respectively. Also, we set

$$
\hat{y}_{i}=y_{i} \prod_{j=1}^{n} x_{j}^{b_{j i}} \in \mathcal{F}, \quad i=1, \ldots, n
$$

as before, and call them the $\hat{y}$-variables of a seed $(B, x, y, z)$.

(2). For any seed $(B, x, y, z)$ and any $k=1, \ldots, n$, we define a new seed $\left(B^{\prime}, x^{\prime}, y^{\prime}, z^{\prime}\right)=\mu_{k}(B, x, y, z)$, called the mutation of $(B, x, y, z)$ at $k$, as follows:

$$
\begin{aligned}
b_{i j}^{\prime} & = \begin{cases}-b_{i j} & i=k \text { or } j=k \\
b_{i j}+d_{k}\left(\left[-b_{i k}\right]_{+} b_{k j}+b_{i k}\left[b_{k j}\right]_{+}\right) & i, j \neq k,\end{cases} \\
x_{i}^{\prime} & = \begin{cases}x_{k}^{-1}\left(\prod_{j=1}^{n} x_{j}^{\left[-b_{j k}\right]_{+}}\right)^{d_{k}} \frac{P_{d_{k}, z_{k}}\left(\hat{y}_{k}\right)}{\left.P_{d_{k}, z_{k}}\left(y_{k}\right)\right|_{\mathbb{P}}} & i=k \\
x_{i} & i \neq k,\end{cases} \\
y_{i}^{\prime} & = \begin{cases}y_{k}^{-1} & i=k \\
y_{i}\left(y_{k}^{\left[b_{k i}\right]_{+}}\right)^{d_{k}}\left(\left.P_{d_{k}, z_{k}}\left(y_{k}\right)\right|_{\mathbb{P}}\right)^{-b_{k i}} & i \neq k .\end{cases} \\
z_{i, s}^{\prime} & =z_{i, d_{i}-s} .
\end{aligned}
$$

Here and elsewhere, for the $z$-variables $z=\left(z_{i, s}\right)_{i=1, \ldots, n ; s=0, \ldots, d_{i}}$, we set

$$
z_{k}:=\left(z_{k, s}\right)_{s=0}^{d_{k}},
$$

and

$$
P_{d_{k}, z_{k}}\left(\hat{y}_{k}\right)=\sum_{s=0}^{d_{k}} z_{k, s} \hat{y}_{k}^{s},\left.\quad P_{d_{k}, z_{k}}\left(y_{k}\right)\right|_{\mathbb{P}}=\bigoplus_{s=0}^{d_{k}} z_{k, s} y_{k}^{s} .
$$

The following facts can be easily checked:

(1) The mutation $\mu_{k}$ is an involution, i.e., $\mu_{k}\left(\mu_{k}(B, x, y, z)\right)=(B, x, y, z)$.

(2) If $R$ is a skew-symmetrizer of $B$, then $R$ is also a skew-symmetrizer of $B^{\prime}$.

(3) The $\hat{y}$-variables in (4.1) transform in $\mathcal{F}$ as the $y$-variables, namely,

$$
\hat{y}_{i}^{\prime}= \begin{cases}\hat{y}_{k}^{-1} & i=k \\ \hat{y}_{i}\left(\hat{y}_{k}^{\left[b_{k i}\right]_{+}}\right)^{d_{k}}\left(P_{d_{k}, z_{k}}\left(\hat{y}_{k}\right)\right)^{-b_{k i}} & i \neq k .\end{cases}
$$




\subsection{Dilogarithm identities.}

Let $n$ and $d=\left(d_{i}\right)_{i=1}^{n}$ be the ones in Section 4.1. Let $\mathbb{Q}_{+}(y, z)$ be the universal semifield with generators (formal commutative variables) $y=\left(y_{i}\right)_{i=1}^{n}$ and $z=$ $\left(z_{i, s}\right)_{i=1, \ldots, n ; s=0, \ldots, d_{i}}$ as defined in Example $2.4(2)$. However, in accordance with our situation, $z_{i, 0}$ and $z_{i, d_{i}}(i=1, \ldots, n)$ are specialized to the identity element 1 . For example, in the case $d=(1, \ldots, 1)$, we have $\mathbb{Q}_{+}(y, z)=\mathbb{Q}_{+}(y)$.

From now on we specialize the coefficient semifield $\mathbb{P}$ in Definition 4.1 as $\mathbb{P}=$ $\mathbb{Q}_{+}(y, z)$. Let us choose a seed $(B, x, y, z)$, where $B$ and $x$ are arbitrary, but we especially choose $y$ and $z$ to be the generators $y$ and $z$ of $\mathbb{Q}_{+}(y, z)$. Let us call $(B, x, y, z)$ the initial seed, and consider a sequence of mutations starting from it:

$$
\begin{aligned}
(B, x, y, z)= & (B[1], x[1], y[1], z[1]) \stackrel{\mu_{k_{1}}}{\rightarrow}(B[2], x[2], y[2], z[2]) \stackrel{\mu_{k_{2}}}{\rightarrow} \cdots \\
& \cdots \stackrel{\mu_{k_{m}}}{\rightarrow}(B[m+1], x[m+1], y[m+1], z[m+1]) .
\end{aligned}
$$

Let $R=\operatorname{diag}\left(r_{1}, \ldots, r_{n}\right)$ be a common skew-symmetrizer of $B[1], \ldots, B[m+1]$, and let $r$ be the least common multiple of $r_{1}, \ldots, r_{n}$. We set $\tilde{r}_{i}=r / r_{i} \in \mathbb{Z}_{+}$.

DeFinition 4.2. We say that the sequence (4.9) is $\sigma$-periodic for a permutation $\sigma$ of $\{1, \ldots, n\}$ if

$$
b_{\sigma(i) \sigma(j)}[m+1]=b_{i j}, \quad x_{\sigma(i)}[m+1]=x_{i}, \quad y_{\sigma(i)}[m+1]=y_{i}, \quad(i, j=1, \ldots, n) .
$$

Proposition 4.3. If the sequence (4.9) is $\sigma$-periodic, then we have

$$
r_{\sigma(i)}=r_{i}, \quad(i=1, \ldots, n) .
$$

Proof. Without losing generality, one can assume that $B=B[1]$ is decomposed into a block diagonal form such that each block is indecomposable. By (2.20), mutations preserve the block diagonal form. Moreover, by (2.22) and the assumption $y_{\sigma(i)}[m+$ $1]=y_{i}, \sigma$ only permutes the indices in the same block. On the other hand, for each indecomposable block $\left(b_{i j}\right)_{i, j=p}^{q}$, its skew-symmetrizer is unique up to a multiplicative constant. In particular, there is a unique minimal skew-symmetrizer $\operatorname{diag}\left(r_{p}^{\prime}, \ldots, r_{q}^{\prime}\right)$ of the block. Then, by the assumption $b_{\sigma(i) \sigma(j)}[m+1]=b_{i j}, \operatorname{diag}\left(r_{\sigma(p)}^{\prime}, \ldots, r_{\sigma(q)}^{\prime}\right)$ is also the minimal skew-symmetrizer of the block. Therefore, we have $r_{\sigma(i)}^{\prime}=r_{i}^{\prime}(i=p, \ldots, q)$. Thus, $r_{\sigma(i)}=r_{i}(i=p, \ldots, q)$ holds.

The following fact connects generalized cluster algebras and dilogarithm identities of higher degree.

THEOREM 4.4 (cf. Theorem 2.7). Suppose that the sequence (4.9) is $\sigma$-periodic for some permutation $\sigma$. Then, it satisfies the following "constancy condition" in the wedge product $\bigwedge^{2} \mathbb{Q}_{+}(y, z)$, where we regard $\mathbb{Q}_{+}(y, z)$ as a multiplicative abelian group:

$$
\sum_{t=1}^{m} \tilde{r}_{k_{t}}\left(y_{k_{t}}[t] \wedge P_{d_{k_{t}}, z_{k_{t}}[t]}\left(y_{k_{t}}[t]\right)\right)=0 .
$$


Our proof of Theorem 4.4 relies on some detailed results on the $y$-variables obtained in $[\mathbf{N a k 1 5 b}$, and it will be given in Section 5 .

Admitting Theorem 4.4, we give the first half of the dilogarithm identity of higher degree associated with any period of seeds.

TheOREm 4.5 (cf. Theorem 2.8). Suppose that the sequence (4.9) is $\sigma$-periodic for some permutation $\sigma$. Let

$$
\varphi: \mathbb{Q}_{+}(y, z) \rightarrow \mathbb{R}_{+}
$$

be any semifield homomorphism such that $\varphi\left(z_{i}\right)=\left(\varphi\left(z_{i, s}\right)\right)_{s=0}^{d_{i}}$ satisfies the generic condition (3.2) for any $i=1, \ldots, n$. Then, the following sum only depend on the images of the $z$-variables $\varphi\left(z_{i, s}\right)$, and does not depend on the images of the $y$-variables $\varphi\left(y_{i}\right)$ :

$$
\sum_{t=1}^{m} \tilde{r}_{k_{t}} \tilde{L}_{d_{k_{t}}, \varphi\left(z_{k_{t}}[t]\right)}\left(\varphi\left(y_{k_{t}}[t]\right)\right) \text {. }
$$

Proof. The argument is standard (e.g., the proof of [Nak11b, Theorem 6.4]). Suppose that there are two semifield homomorphisms $\varphi_{0}$ and $\varphi_{1}$ from $\mathbb{Q}_{+}(y, z)$ to $\mathbb{R}_{+}$ such that $\varphi_{0}\left(z_{i, s}\right)=\varphi_{1}\left(z_{i, s}\right)$. Then, we can interpolate them by a family of semifield homomorphisms $\varphi_{u}(u \in[0,1])$,

$$
\begin{aligned}
\varphi_{u}: \mathbb{Q}_{+}(y, z) & \rightarrow \quad \mathbb{R}_{+} \\
y_{i} & \mapsto(1-u) \varphi_{0}\left(y_{i}\right)+u \varphi_{1}\left(y_{i}\right) \\
z_{i, s} & \mapsto \quad \varphi_{0}\left(z_{i, s}\right)=\varphi_{1}\left(z_{i, s}\right) .
\end{aligned}
$$

Let us introduce positive-real-valued and differentiable functions $Y_{t} \in \mathcal{C}(t=1, \ldots, m)$ on the interval $[0,1]$ defined by

$$
Y_{t}(u)=\varphi_{u}\left(y_{k_{t}}[t]\right)
$$

Applying the family of homomorphisms $\varphi_{u}(u \in[0,1])$ to $(4.12)$, we have the constancy condition in $\bigwedge^{2} \mathcal{C}$ :

$$
\sum_{t=1}^{m} \tilde{r}_{k_{t}}\left(Y_{t} \wedge P_{d_{k}, \varphi_{0}\left(z_{k_{t}}[t]\right)}\left(Y_{t}\right)\right)=0,
$$

where we used the fact that $\varphi_{u}\left(z_{k_{t}}[t]\right)=\varphi_{0}\left(z_{k_{t}}[t]\right)$ for any $u \in[0,1]$. Then, by Theorem 3.2 , the dilogarithm sum

$$
\sum_{t=1}^{m} \tilde{r}_{k_{t}} \tilde{L}_{d_{t}, \varphi_{0}\left(z_{k_{t}}[t]\right)}\left(Y_{t}(u)\right)
$$

is constant as a function of $u \in[0,1]$. In particular, setting $u=0$ and 1 , we have

$$
\sum_{t=1}^{m} \tilde{r}_{k_{t}} \tilde{L}_{d_{t}, \varphi_{0}\left(z_{k_{t}}[t]\right)}\left(\varphi_{0}\left(y_{k_{t}}[t]\right)\right)=\sum_{t=1}^{m} \tilde{r}_{k_{t}} \tilde{L}_{d_{t}, \varphi_{0}\left(z_{k_{t}}[t]\right)}\left(\varphi_{1}\left(y_{k_{t}}[t]\right)\right),
$$


which is the desired result.

The second half of the dilogarithm identity of higher degree is about the constant value of (4.14). To describe it, we introduce the semifield homomorphism (tropicalization map)

$$
\begin{aligned}
\pi: \mathbb{Q}_{+}(y, z) & \rightarrow \operatorname{Trop}(y, z) \\
y_{i} & \mapsto y_{i} \\
z_{i, s} & \mapsto z_{i, s} .
\end{aligned}
$$

Here, $\operatorname{Trop}(y, z)$ is the tropical semifield with generators $y=\left(y_{i}\right)_{i=1}^{n}$ and $z=$ $\left(z_{i, s}\right)_{i=1, \ldots, n ; s=0, \ldots, d_{i}}$ as defined in Example $2.4(3)$, but again $z_{i, 0}$ and $z_{i, d_{i}}(i=1, \ldots, n)$ are specialized to the identity element 1.

We then apply the map $\pi$ to each $y$-variable $y_{i}[t]$ in (4.9). It is known that the image $\pi\left(y_{i}[t]\right)$ does not depend on $z$-variables $z$ [Nak15 b, Lemma 3.6]; thus, it is expressed as

$$
\pi\left(y_{i}[t]\right)=\prod_{j=1}^{n} y_{j}^{c_{j i}[t]}
$$

Thus, we have a family of square matrices $C[t]=\left(c_{i j}[t]\right)_{i, j=1}^{n}$ for $t=1, \ldots, m+1$, which are called the $C$-matrices for the sequence (4.9). Alternatively, they can be directly defined through the following system of recursion relations [Nak15b, Propostition 3.8]:

(initial condition)

$$
c_{i j}[1]=\delta_{i j},
$$

(recursion relation)

$$
c_{i j}[t+1]= \begin{cases}-c_{i k_{t}}[t] & j=k_{t} \\ c_{i j}[t]+d_{k_{t}}\left(\left[-c_{i k_{t}}[t]\right]_{+} b_{k_{t} j}[t]+c_{i k_{t}}[t]\left[b_{k_{t} j}[t]\right]_{+}\right) & j \neq k_{t} .\end{cases}
$$

The $i$ th column vector $c_{i}[t]=\left(c_{j i}[t]\right)_{j=1}^{n}$ of the matrix $C[t]$ is called the $c$-vector of $y[t]_{i}$. By the definition of (4.21), it is the "exponent vector" of the tropical $y$-variable $\pi\left(y_{i}[t]\right)$.

It is known that the sign-coherence property of the $c$-vectors still holds for generalized cluster algebras.

THEOREM 4.6 ([Nak15b, Theorem 3.20]). Each c-vector $c_{i}[t]$ is a nonzero vector, and all its components are either nonnegative or nonpositive.

Accordingly, we set the tropical sign $\varepsilon\left(y_{i}[t]\right) \in\{ \pm 1\}$ of $y_{i}[t]$ as 1 (resp. -1 ) if all components of $c_{i}[t]$ is nonnegative (resp. nonpositive). For simplicity, let us write

$$
\varepsilon_{t}=\varepsilon\left(y_{k_{t}}[t]\right) .
$$

Now, continuing from Theorem 4.5, we can state the second half of the dilogarithm identity of higher degree. 
ThEOREM 4.7 (cf. Theorem 2.11). Under the assumption of Theorem 4.5, we have the following equality for any choice of $\varphi$ in (4.13):

$$
\sum_{t=1}^{m} \tilde{r}_{k_{t}} \tilde{L}_{d_{k_{t}}, \varphi\left(z_{k_{t}}[t]\right)}\left(\varphi\left(y_{k_{t}}[t]\right)\right)=\sum_{t=1}^{m} \tilde{r}_{k_{t}} \frac{1-\varepsilon_{t}}{2} \tilde{L}_{d_{k_{t}}, \varphi\left(z_{k_{t}}[t]\right)}(\infty) .
$$

Proof. Again, the argument is standard (e.g., the proof of [Nak11b, Theorem 6.4]). Let $\varphi$ be any such semifield homomorphism. Then, we consider a family of semifield homomorphism $\varphi_{u}(u \in(0,1])$ as follows:

$$
\begin{aligned}
\varphi_{u}: \mathbb{Q}_{+}(y, z) & \rightarrow \mathbb{R}_{+} \\
y_{i} & \mapsto u \varphi\left(y_{i}\right) \\
z_{i, s} & \mapsto \varphi\left(z_{i, s}\right) .
\end{aligned}
$$

First, we claim the following behavior of $y$-variables in the limit $u \rightarrow 0$ :

$$
\lim _{u \rightarrow 0} \varphi_{u}\left(y_{k_{t}}[t]\right)= \begin{cases}0 & \varepsilon_{t}=1 \\ \infty & \varepsilon_{t}=-1\end{cases}
$$

This follows from the forthcoming expression of the $y$-variables (5.3), together with the sign-coherence property in Theorem 4.6 and the fact that all polynomials $F_{j}[t](y, z)$ in (5.3) have the constant term 1 [Nak15b, Proposition 3.19].

On the other hand, by Theorem 4.5, one can replace $\varphi\left(y_{k_{t}}[t]\right)$ in the left side of (4.25) with $\varphi_{u}\left(y_{k_{t}}[t]\right)$ for any $u \in(0,1]$ without changing the sum therein. Then, by taking the limit $u \rightarrow 0$, we obtain the right-hand side of (4.25) thanks to (4.27).

Using (3.9), we also have an alternative form of the identity (4.25), which is constantterm free.

TheOREM 4.8 (cf. Theorem 2.12). The identity (4.25) is equivalent to the following one:

$$
\sum_{t=1}^{m} \varepsilon_{t} \tilde{r}_{k_{t}} \tilde{L}_{d_{k_{t}}, \varphi\left(\left(z_{k_{t}}[t]\right)^{\circ}\right)}\left(\varphi\left(\left(y_{k_{t}}[t]\right)^{\varepsilon_{t}}\right)\right)=0
$$

where

$$
\left(z_{k_{t}}[t]\right)^{\circ}= \begin{cases}z_{k_{t}}[t] & \varepsilon_{t}=1 \\ \left(z_{k_{t}}[t]\right)^{*} & \varepsilon_{t}=-1 .\end{cases}
$$

REMARK 4.9. One can force to set some of $\varphi\left(z_{i, s}\right)$ to be 0 , and Theorems 4.7 and 4.8 still hold by continuity.

\section{Proof of Theorem 4.4.}

In this section we give a proof of Theorem 4.4, which is the core of this paper. The proof here uses the same argument in the "second proof" of [Nak11b, Proposition 6.3] 
therein, whose idea originates in [FG09a, Proposition 6.3] and [FG09b, Proposition 2.14]. Interestingly, even though the statement of Theorem 4.4 only involves $y$ - and $z$ variables, the proof requires $F$-polynomials as well, which are the specializations of the accompanying $x$-variables.

Let us recall the notion of the F-polynomials for generalized cluster algebras $[\mathbf{N a k 1 5 b}]$ in our context.

Definition 5.1. Let us consider the sequence (4.9). Apply the tropicalization map of (4.20) to all $y$-variables involving in the mutation of $x$-variables (4.3). Then, it is known [Nak15b, Proposition 3.3] that the resulting $x$-variable $x_{i}[t]$ is expressed as a Laurent polynomial $X_{i}[t](x, y, z) \in \mathbb{Z}\left[x^{ \pm 1}, y, z\right]$. By specializing $x_{1}=\ldots=x_{n}=1$ in $X_{i}[t](x, y, z)$, we obtain a polynomial $F_{i}[t](y, z) \in \mathbb{Z}[y, z]$, which is called the $F$-polynomial of $x_{i}[t]$.

We use the following known properties of the $F$-polynomials, which generalize the ones for ordinary cluster algebras by [FZ07].

Proposition 5.2. (1). ([Nak15b, Proposition 3.12]) The F-polynomials satisfy the following system of recursion relations:

(initial condition)

$$
F_{i}[1](y, z)=1
$$

(recursion relation)

$$
F_{i}[t+1](y, z)=\left\{\begin{array}{cl}
F_{k_{t}}[t](y, z)^{-1}\left(\prod_{j=1}^{n} y_{j}^{\left[-c_{j k_{t}}[t]\right]_{+}} F_{j}[t](y, z)^{\left[-b_{j k_{t}}[t]\right]_{+}}\right)^{d_{k_{t}}} & \\
\quad \times P_{d_{k_{t}}, z_{k_{t}}[t]}\left(\prod_{j=1}^{n} y_{j}^{c_{j k_{t}}[t]} F_{j}[t](y, z)^{b_{j k_{t}}[t]}\right) & i=k_{t} \\
F_{i}[t](y, z) & i \neq k_{t} .
\end{array}\right.
$$

(2). ([Nak15b, Theorem 3.22]) The following equality holds:

(Separation formula)

$$
y_{i}[t]=\prod_{j=1}^{n} y_{j}^{c_{j i}[t]} F_{j}[t](y, z)^{b_{j i}[t]} .
$$

We note that due to the above recursion, $F_{i}[t](y, z)$ are also viewed as elements in $\mathbb{Q}_{+}(y, z)$.

We also need the following periodicity property of the $F$-polynomials.

Proposition 5.3. Suppose that the sequence (4.9) is $\sigma$-periodic for some permutation $\sigma$. Then, the F-polynomials obey the same periodicity, i.e.,

$$
F_{\sigma(i)}[m+1](y, z)=F_{i}[1](y, z)=1 .
$$


Therefore, $F_{i}[m+1](y, z)=1$ for any $i=1, \ldots, n$.

Proof. This is because the $F$-polynomials are defined by the tropicalization and specialization from the $x$-variables in (4.9).

To each seed $\Sigma[t]=(B[t], x[t], y[t], z[t])(t=1, \ldots, m+1)$ in the sequence (4.9), we attach the following element in $\bigwedge^{2} \mathbb{Q}_{+}(y, z)$ :

$$
V[t]:=\sum_{i=1}^{n} \tilde{r}_{i}\left(F_{i}[t] \wedge y_{i}[t]\right)+\frac{1}{2} \sum_{i, j=1}^{n} b_{i j}[t] \tilde{r}_{j}\left(F_{i}[t] \wedge F_{j}[t]\right) .
$$

Note that $V[1]=0$ due to the initial condition (5.1).

The next result is crucial in our proof of Theorem 4.4.

LEMMA 5.4. The following equality holds:

$$
V[t+1]-V[t]=\tilde{r}_{k_{t}}\left(y_{k_{t}}[t] \wedge P_{d_{k_{t}}, z_{k_{t}}[t]}\left(y_{k_{t}}[t]\right)\right) .
$$

Proof. We prove it by the direct and straightforward calculation. To make the calculation a little more transparent, we separate the quantity $V[t]$ in (5.5) into two parts,

$$
V_{1}[t]=\sum_{i=1}^{n} \tilde{r}_{i}\left(F_{i}[t] \wedge y_{i}[t]\right), \quad V_{2}[t]=\frac{1}{2} \sum_{i, j=1}^{n} b_{i j}[t] \tilde{r}_{j}\left(F_{i}[t] \wedge F_{j}[t]\right)
$$

and calculate the difference $V_{i}[t+1]-V_{i}[t]$ separately. After a careful calculation, we obtain the following results.

$$
\begin{aligned}
V_{1}[t+1]-V_{1}[t]= & \tilde{r}_{k_{t}}\left(\left(\prod_{i=1}^{n} F_{i}[t]^{b_{i k_{t}}[t]}\right) \wedge P_{d_{k_{t}}, z_{k_{t}}[t]}\left(y_{k_{t}}[t]\right)\right) \\
& +d_{k_{t}} \tilde{r}_{k_{t}}\left(y_{k_{t}}[t] \wedge\left(\prod_{i=1}^{n} y_{i}^{\left[-c_{i k_{t}}[t]\right]_{+}}\right)\right) \\
& +\tilde{r}_{k_{t}}\left(y_{k_{t}}[t] \wedge P_{d_{k_{t}}, z_{k_{t}}[t]}\left(y_{k_{t}}[t]\right)\right), \\
V_{2}[t+1]-V_{2}[t]= & -\tilde{r}_{k_{t}}\left(\left(\prod_{i=1}^{n} F_{i}[t]^{b_{i k_{t}}[t]}\right) \wedge P_{d_{k_{t}}, z_{k_{t}}[t]}\left(y_{k_{t}}[t]\right)\right) \\
& -d_{k_{t}} \tilde{r}_{k_{t}}\left(\left(\prod_{i=1}^{n}\left(F_{i}[t]\right)^{b_{i k_{t}}[t]}\right) \wedge\left(\prod_{i=1}^{n} y_{i}^{\left.\left[-c_{i k_{t}}[t]\right]_{+}\right)}\right) .\right.
\end{aligned}
$$

To obtain them, we used (4.2), (4.4), (5.2), and also the skew-symmetric property

$$
b_{i j}[t] \tilde{r}_{j}=-b_{j i}[t] \tilde{r}_{i}
$$

Summing up (5.8) and (5.9) and using (5.3), we have 


$$
\begin{aligned}
V[t+1]-V[t]= & d_{k_{t}} \tilde{r}_{k_{t}}\left(\left(\prod_{i=1}^{n} y_{i}^{c_{i k_{t}}[t]}\right) \wedge\left(\prod_{i=1}^{n} y_{i}^{\left[-c_{i k_{t}}[t]\right]_{+}}\right)\right) \\
& +\tilde{r}_{k_{t}}\left(y_{k_{t}}[t] \wedge P_{d_{k_{t}}, z_{k_{t}}[t]}\left(y_{k_{t}}[t]\right)\right) .
\end{aligned}
$$

Then, the proof of the lemma completes by showing the first term in the right-hand side of (5.11) vanishes. Indeed, using the equality $a=[a]_{+}-[-a]_{+}$, we have

$$
\left(\prod_{i=1}^{n} y_{i}^{c_{i k_{t}}[t]}\right) \wedge\left(\prod_{i=1}^{n} y_{i}^{\left[-c_{i k_{t}}[t]\right]_{+}}\right)=\left(\prod_{i=1}^{n} y_{i}^{\left[c_{i k_{t}}[t]\right]_{+}}\right) \wedge\left(\prod_{i=1}^{n} y_{i}^{\left[-c_{i k_{t}}[t]\right]_{+}}\right) \text {. }
$$

Then, either the first or the second component in the right-hand side of (5.12) is 1 due to the sign-coherence property of the $c$-vectors in Theorem 4.6. Thus, the right-hand side of (5.12) vanishes as desired.

Let us complete the proof of Theorem 4.4.

Proof of Theorem 4.4. Due to the assumption of the periodicity (4.10) and the resulting periodicities of the skew-symmetrizer (4.11) and the $F$-polynomials (5.4), we have the periodicity of $V[t]$, i.e.,

$$
V[m+1]=V[1]=0 .
$$

On the other hand, by Lemma 5.4, we have

$$
V[m+1]=V[m+1]-V[1]=\sum_{t=1}^{m} \tilde{r}_{k_{t}}\left(y_{k_{t}}[t] \wedge P_{d_{k_{t}}, z_{k_{t}}[t]}\left(y_{k_{t}}[t]\right)\right) .
$$

Combining (5.13) and (5.14), we obtain the constancy condition (4.12).

\section{Examples.}

Here we provide three examples of periodicities in generalized cluster algebras which are not regarded as the periodicities in ordinary cluster algebras. In all of them the permutation $\sigma$ in Definition 4.2 is the trivial one. So far, we do not know any example of a periodicity with a nontrivial permutation $\sigma$ which is not regarded as a periodicity in an ordinary cluster algebra. (For ordinary cluster algebras there are plenty examples of periodicities with nontrivial permutations. See e.g. $\left[\mathbf{I I K}^{+} \mathbf{1 3}\right.$, Theorem 7.1].)

\subsection{Involution periodicity.}

Let $(B, x, y, z)$ be any seed with any rank $n$ and any mutation degree $d$. Due to the involution property of the mutation $\mu_{k}$, the mutation sequence

$$
(B, x, y, z)=(B[1], x[1], y[1], z[1]) \stackrel{\mu_{\xi}}{\rightarrow}(B[2], x[2], y[2], z[2]) \stackrel{\mu_{k}}{\rightarrow}(B[3], x[3], y[3], z[3]) .
$$

is periodic, i.e., $\sigma$-periodic with $\sigma=\mathrm{id}$. The data for the associated dilogarithm identity is given as follows: 


$$
\begin{gathered}
y_{k}[1]=y_{k}, \quad y_{k}[2]=y_{k}^{-1}, \\
\varepsilon_{1}=1, \quad \varepsilon_{2}=-1, \\
z_{k}[1]=z_{k}, \quad z_{k}[2]=z_{k}^{*} .
\end{gathered}
$$

Thus, the dilogarithm identity in the form (4.25) is

$$
\tilde{r}_{k} \tilde{L}_{d_{k}, z_{k}}\left(y_{k}\right)+\tilde{r}_{k} \tilde{L}_{d_{k}, z_{k}^{*}}\left(y_{k}^{-1}\right)=\tilde{r}_{k} \tilde{L}_{d_{k}, z_{k}^{*}}(\infty),
$$

where for notational simplicity we omit the evaluation homomorphism $\varphi: \mathbb{Q}_{+}(y, z) \rightarrow \mathbb{R}_{+}$ by identifying $y_{k}$ and $z_{k}$ with their images in $\mathbb{R}_{+}$by $\varphi$. The identity (6.5) coincides with (3.4). Meanwhile, the dilogarithm identity in the form (4.28) becomes trivial.

$$
\tilde{r}_{k} \tilde{L}_{d_{k}, z_{k}}\left(y_{k}\right)-\tilde{r}_{k} \tilde{L}_{d_{k}, z_{k}}\left(y_{k}\right)=0 .
$$

\subsection{Six term relation for type $B_{2} / C_{2}$.}

Let $n=2$ and $d=(2,1)$. We consider an initial seed $(B, x, y, z)$ with

$$
B=\left(\begin{array}{cc}
0 & -1 \\
1 & 0
\end{array}\right), \quad z_{1}=(1, \alpha, 1), \quad z_{2}=(1,1) .
$$

We choose $r_{1}=r_{2}=1$, so that $\tilde{r}_{1}=\tilde{r}_{2}=1$. Then, the following sequence of alternative mutations is known to be periodic [Nak15b, Section 2.3]:

$$
\begin{aligned}
(B, x, y, z)=(B[1], x[1], y[1], z[1]) & \stackrel{\mu_{1}}{\rightarrow}(B[2], x[2], y[2], z[2]) \stackrel{\mu_{2}}{\rightarrow} \\
& \cdots \stackrel{\mu_{2}}{\rightarrow}(B[7], x[7], y[7], z[7]) .
\end{aligned}
$$

The data for the associated dilogarithm identity is given as follows:

$$
\begin{aligned}
& y_{1}[1]=y_{1}, \quad y_{2}[2]=y_{2}\left(1+\alpha y_{1}+y_{1}^{2}\right), \quad y_{1}[3]=y_{1}^{-1}\left(1+y_{2}+\alpha y_{1} y_{2}+y_{1}^{2} y_{2}\right), \\
& y_{2}[4]=y_{1}^{-2} y_{2}^{-1}\left(1+2 y_{2}+y_{2}^{2}+\alpha y_{1} y_{2}+\alpha y_{1} y_{2}^{2}+y_{1}^{2} y_{2}^{2}\right), \\
& y_{1}[5]=y_{1}^{-1} y_{2}^{-1}\left(1+y_{2}\right), \quad y_{2}[6]=y_{2}^{-1} \\
& \varepsilon_{1}=\varepsilon_{2}=1, \quad \varepsilon_{3}=\cdots=\varepsilon_{6}=-1, \\
& z_{1}[1]=z_{1}[3]=z_{1}[5]=z_{1}, \quad z_{2}[2]=z_{2}[4]=z_{2}[6]=z_{2} .
\end{aligned}
$$

Then, the dilogarithm identity in the form (4.28) is explicitly written as follows:

$$
\begin{aligned}
& \tilde{L}_{2, z_{1}}\left(y_{1}\right)+\tilde{L}\left(y_{2}\left(1+\alpha y_{1}+y_{1}^{2}\right)\right)-\tilde{L}_{2, z_{1}}\left(y_{1}\left(1+y_{2}+\alpha y_{1} y_{2}+y_{1}^{2} y_{2}\right)^{-1}\right) \\
& -\tilde{L}\left(y_{1}^{2} y_{2}\left(1+2 y_{2}+y_{2}^{2}+\alpha y_{1} y_{2}+\alpha y_{1} y_{2}^{2}+y_{1}^{2} y_{2}^{2}\right)^{-1}\right) \\
& -\tilde{L}_{2, z_{1}}\left(y_{1} y_{2}\left(1+y_{2}\right)^{-1}\right)-\tilde{L}\left(y_{2}\right)=0,
\end{aligned}
$$

where $\tilde{L}(x):=\tilde{L}(x)_{1, z_{2}}=L(x /(1+x))$ for the ordinary Rogers dilogarithm $L(x)$.

\subsection{Eight term relation for type $\boldsymbol{G}_{\mathbf{2}}$.}

Let $n=2$ and $d=(3,1)$. We consider an initial seed $(B, x, y, z)$ with 


$$
B=\left(\begin{array}{cc}
0 & -1 \\
1 & 0
\end{array}\right), \quad z_{1}=(1, \alpha, \beta, 1), \quad z_{2}=(1,1)
$$

We choose $r_{1}=r_{2}=1$, so that $\tilde{r}_{1}=\tilde{r}_{2}=1$. Then, the following sequence of alternative mutations is known to be periodic [NR16, Example 3.7]:

$$
\begin{aligned}
(B, x, y, z)=(B[1], x[1], y[1], z[1]) & \stackrel{\mu_{\rightarrow}}{\rightarrow}(B[2], x[2], y[2], z[2]) \stackrel{\mu_{2}}{\rightarrow} \\
& \cdots \stackrel{\mu_{2}}{\rightarrow}(B[9], x[9], y[9], z[9]) .
\end{aligned}
$$

The data for the associated dilogarithm identity is given as follows:

$$
\begin{aligned}
& y_{1}[1]=y_{1}, \quad y_{2}[2]=y_{2}\left(1+\alpha y_{1}+\beta y_{1}^{2}+y_{1}^{3}\right), \\
& y_{1}[3]=y_{1}^{-1}\left(1+y_{2}+\alpha y_{1} y_{2}+\beta y_{1}^{2} y_{2}+y_{1}^{3} y_{2}\right), \\
& y_{2}[4]=y_{1}^{-3} y_{2}^{-1}\left(1+3 y_{2}+3 y_{2}^{2}+y_{2}^{3}+2 \alpha y_{1} y_{2}+4 \alpha y_{1} y_{2}^{2}+2 \alpha y_{1} y_{2}^{3}\right. \\
& +\beta y_{1}^{2} y_{2}+\alpha^{2} y_{1}^{2} y_{2}^{2}+3 \beta y_{1}^{2} y_{2}^{2}+\alpha^{2} y_{1}^{2} y_{2}^{3}+2 \beta y_{1}^{2} y_{2}^{3} \\
& +\alpha \beta y_{1}^{3} y_{2}^{2}+2 \alpha \beta y_{1}^{3} y_{2}^{3}+3 y_{1}^{3} y_{2}^{2}+2 y_{1}^{3} y_{2}^{3} \\
& \left.+\alpha y_{1}^{4} y_{2}^{2}+2 \alpha y_{1}^{4} y_{2}^{3}+\beta^{2} y_{1}^{4} y_{2}^{3}+2 \beta y_{1}^{5} y_{2}^{3}+y_{1}^{6} y_{2}^{3}\right), \\
& y_{1}[5]=y_{1}^{-2} y_{2}^{-1}\left(1+2 y_{2}+y_{2}^{2}+\alpha y_{1} y_{2}+\alpha y_{1} y_{2}^{2}+\beta y_{1}^{2} y_{2}^{2}+y_{1}^{3} y_{2}^{2}\right) \text {, } \\
& y_{2}[6]=y_{1}^{-3} y_{2}^{-2}\left(1+3 y_{2}+3 y_{2}^{2}+y_{2}^{3}+\alpha y_{1} y_{2}+2 \alpha y_{1} y_{2}^{2}+\alpha y_{1} y_{2}^{3}\right. \\
& \left.+\beta y_{1}^{2} y_{2}^{2}+\beta y_{1}^{2} y_{2}^{3}+y_{1}^{3} y_{2}^{3}\right), \\
& y_{1}[7]=y_{1}^{-1} y_{2}^{-1}\left(1+y_{2}\right), \quad y_{2}[8]=y_{2}^{-1} \text {, } \\
& \varepsilon_{1}=\varepsilon_{2}=1, \quad \varepsilon_{3}=\cdots=\varepsilon_{8}=-1 \text {, } \\
& z_{1}[1]=z_{1}[5]=z_{1}, \quad z_{1}[3]=z_{1}[7]=z_{1}^{*}, \quad z_{2}[2]=z_{2}[4]=z_{2}[6]=z_{2}[8]=z_{2} .
\end{aligned}
$$

Then, the dilogarithm identity in the form (4.28) is explicitly written as follows:

$$
\begin{aligned}
& \tilde{L}_{3, z_{1}}\left(y_{1}\right)+\tilde{L}\left(y_{2}\left(1+\alpha y_{1}+\beta y_{1}^{2}+y_{1}^{3}\right)\right) \\
& -\tilde{L}_{3, z_{1}}\left(y_{1}\left(1+y_{2}+\alpha y_{1} y_{2}+\beta y_{1}^{2} y_{2}+y_{1}^{3} y_{2}\right)^{-1}\right) \\
& -\tilde{L}\left(y _ { 1 } ^ { 3 } y _ { 2 } \left(1+3 y_{2}+3 y_{2}^{2}+y_{2}^{3}+2 \alpha y_{1} y_{2}+4 \alpha y_{1} y_{2}^{2}+2 \alpha y_{1} y_{2}^{3}\right.\right. \\
& +\beta y_{1}^{2} y_{2}+\alpha^{2} y_{1}^{2} y_{2}^{2}+3 \beta y_{1}^{2} y_{2}^{2}+\alpha^{2} y_{1}^{2} y_{2}^{3}+2 \beta y_{1}^{2} y_{2}^{3} \\
& +\alpha \beta y_{1}^{3} y_{2}^{2}+2 \alpha \beta y_{1}^{3} y_{2}^{3}+3 y_{1}^{3} y_{2}^{2}+2 y_{1}^{3} y_{2}^{3} \\
& \left.\left.+\alpha y_{1}^{4} y_{2}^{2}+2 \alpha y_{1}^{4} y_{2}^{3}+\beta^{2} y_{1}^{4} y_{2}^{3}+2 \beta y_{1}^{5} y_{2}^{3}+y_{1}^{6} y_{2}^{3}\right)^{-1}\right) \\
& -\tilde{L}_{3, z_{1}^{*}}\left(y_{1}^{2} y_{2}\left(1+2 y_{2}+y_{2}^{2}+\alpha y_{1} y_{2}+\alpha y_{1} y_{2}^{2}+\beta y_{1}^{2} y_{2}^{2}+y_{1}^{3} y_{2}^{2}\right)^{-1}\right) \\
& -\tilde{L}\left(y _ { 1 } ^ { 3 } y _ { 2 } ^ { 2 } \left(1+3 y_{2}+3 y_{2}^{2}+y_{2}^{3}+\alpha y_{1} y_{2}+2 \alpha y_{1} y_{2}^{2}+\alpha y_{1} y_{2}^{3}\right.\right. \\
& \left.\left.+\beta y_{1}^{2} y_{2}^{2}+\beta y_{1}^{2} y_{2}^{3}+y_{1}^{3} y_{2}^{3}\right)^{-1}\right) \\
& -\tilde{L}_{3, z_{1}}\left(y_{1} y_{2}\left(1+y_{2}\right)^{-1}\right)-\tilde{L}\left(y_{2}\right)=0 \text {. }
\end{aligned}
$$




\section{A. Derivation of classical dilogarithm identity from quantum one.}

This appendix serves as independent reading.

Here we complete the picture by showing how the classical dilogarithm identity of higher degree in Theorem 4.8 is obtained from its quantum counterpart in [Nak15a, Theorem 4.1]. This is a generalization of the argument in [KN11] for the ordinary cluster algebras with skew-symmetric exchange matrices. (Thus, this presentation is new even for the ordinary cluster algebras with skew-symmetrizable exchange matrices.)

We rely on the saddle point method, which is standard in quantum mechanics (e.g., [Tak08, p. 95]). However, as in [KN11], we stress that the derivation here is only heuristic, and not functional-analytically rigorous; for example, the uniqueness of the solution of the saddle point equations, the specification of the integration contour through the saddle point, and the total validity of the method are not pursued. Nevertheless, we believe that the derivation presented here is useful for the readers. (At least it is better than nothing.)

Here we follow and generalize the calculations especially in Section 4 and Appendix A of $[\mathbf{K N 1 1}]$. Since this is a rather complicated subject, we try to write it in a selfcontained way at a reasonable level, but not completely, and we ask the readers to refer to [KN11] (and also [Nak15a]) for further details.

\section{A.1. Quantum dilogarithms of higher degree.}

For any positive integer $d$, the quantum dilogarithm of degree $d$ with coefficients $z=\left(z_{s}\right)_{s=0}^{d}$, where $z_{0}=z_{d}=1$, is defined as [Nak15a]

$$
\mathbf{\Psi}_{d, z, q}(x)=\prod_{k=0}^{\infty} P_{d, z}\left(q^{2 k+1} x\right)^{-1},
$$

where $P_{d, z}(x)$ is the polynomial in $(3.1)$, whose coefficients $z_{s}$ 's are nonnegative real numbers which satisfy the generic condition (3.2), and $q \in \mathbb{C}$ with $|q|<1$. Then, the power series (A.1) converges for any $x \in \mathbb{C}$. Below let us concentrate on the region $x \geq 0$.

The function $\boldsymbol{\Psi}_{d, z, q}(x)$ is related to the dilogarithm of higher degree $\operatorname{Li}_{2 ; d, z}(x)$ in (3.3) in the asymptotic limit as follows [Nak15a]:

$$
\mathbf{\Psi}_{d, z, q}(x) \sim \exp \left(-\frac{\operatorname{Li}_{2 ; d, z}(-x)}{\log q^{2}}\right), \quad q \rightarrow 1^{-} .
$$

\section{A.2. Quantum $\boldsymbol{Y}$-seed and mutations.}

Let us recall the notions of a quantum $Y$-seed and its mutation in generalized cluster algebras following [Nak15a] with slight modification.

Definition A.1. As in the classical case, first we fix the rank $n$ and the mutation degree $d=\left(d_{i}\right)_{i=1}^{n}$. Then, we consider a triplet $(B, Y, z)$ such that

- $B=\left(b_{i j}\right)_{i, j=1}^{n}$ is a skew-symmetrizable integer matrix of size $n$,

- $Y=\left(Y_{i}\right)_{i=1}^{n}$ is an $n$-tuple of noncommutative formal variables obeying the relation

$$
Y_{i} Y_{j}=q_{j}^{2 b_{j i}} Y_{j} Y_{i}, \quad q_{i}:=q^{r_{i}}
$$


where $R=\operatorname{diag}\left(r_{1}, \ldots, r_{n}\right)$ is a skew-symmetrizer of $B$,

- $z=\left(z_{i, s} \mid i=1, \ldots, n ; s=0, \ldots, d_{i}\right)$ is a collection of commutative formal variables with $z_{i, 0}=z_{i, d_{i}}=1$ for any $i=1, \ldots, n$; furthermore, $z_{i, s}$ 's commute with $Y_{j}$ 's.

We call such $(B, Y, z)$ a quantum $Y$-seed, and call $Y=\left(Y_{i}\right)_{i=1}^{n}$ the quantum $y$-variables of $(B, Y, z)$.

It is convenient to extend the above quantum $y$-variables to a family of noncommutative variables $Y^{\alpha}\left(\alpha \in \mathbb{Z}^{n}\right)$ with the relations

$$
q^{\langle\alpha, \beta\rangle} Y^{\alpha} Y^{\beta}=Y^{\alpha+\beta}, \quad\langle\alpha, \beta\rangle=: \sum_{i, j=1}^{n} \alpha_{i} r_{i} b_{i j} \beta_{j},
$$

where we identify $Y_{i}=Y^{e_{i}}$ for the $i$ th unit vector $e_{i}$ of $\mathbb{Z}^{n}$.

Definition A.2. For any quantum $Y$-seed $(B, Y, z)$ and any $k=1, \ldots, n$, we define a new seed $\left(B^{\prime}, Y^{\prime}, z^{\prime}\right)=\mu_{k}(B, Y, z)$, called the mutation of $(B, Y, z)$ at $k$, as follows:

$$
Y_{i}^{\prime}= \begin{cases}Y_{k}^{-1} & i=k \\ Y^{e_{i}+d_{k}\left[b_{k i}\right]_{+} e_{k}} \prod_{m=1}^{\left|b_{k i}\right|}\left(\sum_{s=0}^{d_{k}} z_{k, s} q_{k}^{-\operatorname{sgn}\left(b_{k i}\right)(2 m-1) s} Y_{k}^{s}\right)^{-\operatorname{sgn}\left(b_{k i}\right)} & i \neq k,\end{cases}
$$

where $\operatorname{sgn}(a)=1,-1,0$ if $a>0, a<0, a=0$, respectively, while $B^{\prime}$ and $z^{\prime}$ are defined by (4.2) and (4.5), respectively.

Indeed, it is easy to check that the following relation holds for the same skewsymmetrizer $R$ :

$$
Y_{i}^{\prime} Y_{j}^{\prime}=q_{j}^{2 b_{j i}^{\prime}} Y_{j}^{\prime} Y_{i}^{\prime}
$$

Again, the mutation $\mu_{k}$ is an involution, i.e., $\mu_{k}\left(\mu_{k}(B, Y, z)\right)=(B, Y, z)$.

REMARK A.3. In [Nak15b] and [Nak15a] a skew-symmetrizer $R$ was introduced, not for the exchange matrix $B$ itself, but for the matrix $D B$. Using this opportunity, let us modify the convention to the one in this paper, which is simpler. For example, Equation (3.2) in [Nak15a] is replaced with (A.3); and Equation (3.22) in [Nak15b] is replaced with

$$
R^{-1}\left(G^{t}\right)^{T} R C^{t}=I
$$

\section{A.3. Quantum dilogarithm identity of higher degree.}

Let us choose any quantum $Y$-seed $(B, Y, z)$ as the initial seed, and consider a sequence of mutations starting from it: 


$$
\begin{aligned}
(B, Y, z)=(B[1], Y[1], z[1]) & \stackrel{\mu_{k_{1}}}{\rightarrow}(B[2], Y[2], z[2]) \stackrel{\mu_{k_{2}}}{\rightarrow} \\
& \cdots \stackrel{\mu_{k_{m}}}{\rightarrow}(B[m+1], Y[m+1], z[m+1]),
\end{aligned}
$$

where we use a common skew-symmetrizer $R=\operatorname{diag}\left(r_{1}, \ldots, r_{n}\right)$ of $B[1], \ldots, B[m+1]$ to define the commutation relation for $Y[t]$ all $t=1, \ldots, m+1$.

Definition A.4. We say that the sequence (A.8) is $\sigma$-periodic for a permutation $\sigma$ of $\{1, \ldots, n\}$ if

$$
b_{\sigma(i) \sigma(j)}[m+1]=b_{i j}, \quad Y_{\sigma(i)}[m+1]=Y_{i}, \quad(i, j=1, \ldots, n) .
$$

Along with the sequence (A.8), let us also consider the sequence (4.9) of the mutations of the classical seed, where the initial exchange matrix $B$ is taken to be common in the both sequences.

Conjecture A.5. The sequence (A.8) is $\sigma$-periodic if and only if the sequence (4.9) is $\sigma$-periodic.

The conjecture is known to be true for the ordinary case $d=(1, \ldots, 1)$ with skewsymmetric exchange matrices, i.e., $r=(1, \ldots, 1)\left(\left[\mathbf{K N 1 1}\right.\right.$, Proposition 3.4] and $\left[\mathbf{I I K}^{+} \mathbf{1 3}\right.$, Theorem 5.1]). However, we do not rely on this conjecture in the rest of the paper.

From now on we specialize the $z$-variables $z$ of the initial seed $(B, Y, z)$ in (A.8) to be real positive numbers such that, for each $i=1, \ldots, n, z_{i}:=\left(z_{i, s}\right)_{s=0}^{d_{i}}$ satisfies the generic condition (3.2).

Theorem A.6 ([Nak15a, Theorem 4.1]). Suppose that the sequence (A.8) is $\sigma$ periodic for some permutation $\sigma$. Then, the following equality holds:

$$
\boldsymbol{\Psi}_{d_{k_{1}}, z_{k_{1}}[1], q_{k_{1}}}\left(Y^{\varepsilon_{1} c_{k_{1}}[1]}\right)^{\varepsilon_{1}} \cdots \boldsymbol{\Psi}_{d_{k_{m}}, z_{k_{m}}[m], q_{k_{m}}}\left(Y^{\varepsilon_{m} c_{k_{m}}[m]}\right)^{\varepsilon_{m}}=1
$$

where $z_{k_{t}}[t]:=\left(z_{k_{t}, s}\right)_{s=0}^{d_{k_{t}}}$ as before, $c_{k_{t}}[t]$ is the c-vector for the sequence (4.9) defined by (4.21), and $\varepsilon_{t}$ is the tropical sign of $c_{k_{t}}[t]$ as in (4.24).

REMARK A.7. The identity (A.10) is called the quantum dilogarithm identity in tropical form in [Nak15a] and proved only for the reciprocal case $z_{i}^{*}=z_{i}$ therein, where the $z$-variables do not mutate. However, it is straightforwardly extended to the nonreciprocal case as above.

We will "derive" the classical dilogarithm identity (4.8) from (A.10) in the limit $q \rightarrow 1$. Note that we cannot simply apply the formula (A.2) to (A.10), since the quantum $y$-variables therein are not real numbers, but noncommutative variables. So, our strategy, which is standard in quantum mechanics, is as follows:

- Step 1. Represent those quantum $y$-variables in (A.10) by operators acting on functions.

- Step 2. Take the expectation value of the left-hand side of (A.10), and express it as an integral. 
- Step 3. Evaluate the integral in the limit $q \rightarrow 1$ by the saddle point method.

This process "magically" transforms the (Euler) dilogarithms of higher degree (3.3) into the Rogers dilogarithms of higher degree (3.12). See (A.65)-(A.69) for a preview.

\section{A.4. Step 1: Operator representation.}

We express the deformation parameter $q$ as

$$
q=e^{\lambda^{2} \hbar \sqrt{-1}},
$$

where $\hbar$ is a positive real number, and $\lambda$ be a complex number sufficiently close to 1 such that $\operatorname{Im} \lambda>0$. Later we will take $\hbar \rightarrow 0$ and $\lambda \rightarrow 1$. (See [KN11, Appendix A.1] for the explanation of introducing the parameter $\lambda$.)

Consider the Hilbert space $L^{2}\left(\mathbb{R}^{n}\right)$. Let $u=\left(u_{i}\right)_{i=1}^{n}$ denote the coordinate of $\mathbb{R}^{n}$. Let $\hat{u}_{i}$ and $\hat{p}_{i}$ be the standard position and momentum operators on $L^{2}\left(\mathbb{R}^{n}\right)$ (densely) defined by

$$
\left(\hat{u}_{i} f\right)(u)=u_{i} f(u), \quad\left(\hat{p}_{i} f\right)(u)=\frac{\hbar}{\sqrt{-1}} \frac{\partial f}{\partial u_{i}}(u), \quad f \in L^{2}\left(\mathbb{R}^{2}\right) .
$$

Thus, we have the commutation relations

$$
\left[\hat{u}_{i}, \hat{u}_{j}\right]=\left[\hat{p}_{i}, \hat{p}_{j}\right]=0, \quad\left[\hat{p}_{i}, \hat{u}_{j}\right]=\frac{\hbar}{\sqrt{-1}} \delta_{i j} .
$$

Let $B$ be the initial exchange matrix of the sequence (A.8). Define

$$
\hat{w}_{i}=\sum_{j=1}^{n} b_{j i} \hat{u}_{j}, \quad \hat{D}_{i}=r_{i} \hat{p}_{i}+\hat{w}_{i}, \quad \hat{Y}_{i}=\exp \left(\lambda \hat{D}_{i}\right) .
$$

Then, we have

$$
\left[\hat{D}_{i}, \hat{D}_{j}\right]=2 \hbar \sqrt{-1} r_{j} b_{j i}, \quad \hat{Y}_{i} \hat{Y}_{j}=q_{j}^{2 b_{j i}} \hat{Y}_{j} \hat{Y}_{i}
$$

Thus, we have a representation of the initial quantum $y$-variables satisfying (A.3). More generally, for any $\alpha \in \mathbb{Z}^{n}$, we define

$$
\hat{Y}^{\alpha}=\exp (\lambda \alpha \hat{D}), \quad \alpha \hat{D}=\sum_{i=1}^{n} \alpha_{i} \hat{D}_{i}
$$

Then,

$$
q^{\langle\alpha, \beta\rangle} \hat{Y}^{\alpha} \hat{Y}^{\beta}=\hat{Y}^{\alpha+\beta}, \quad\langle\alpha, \beta\rangle=\sum_{i, j=1}^{n} \alpha_{i} r_{i} b_{i j} \beta_{j},
$$

Thus, they give a representation of $Y^{\alpha}$ 's in (A.4).

Next we describe the mutations of the quantum $y$-variables. Along with the sequence (A.8) with $\sigma$-periodicity, we introduce a sequence of linear transformations 


$$
\mathbb{R}^{n} \stackrel{\rho_{1}}{\rightarrow} \mathbb{R}^{n} \stackrel{\rho_{2}}{\rightarrow} \cdots \stackrel{\rho_{m}}{\rightarrow} \mathbb{R}^{n} \stackrel{\sigma}{\rightarrow} \mathbb{R}^{n}
$$

where, for $t=1, \ldots, m$,

$$
\begin{aligned}
& \rho_{t}: \mathbb{R}^{n} \rightarrow \mathbb{R}^{n}, \quad\left(u_{i}\right)_{i=1}^{n} \mapsto\left(u_{i}^{\prime}\right)_{i=1}^{n}, \\
& u_{i}^{\prime}= \begin{cases}-u_{k_{t}}+d_{k_{t}} \sum_{j=1}^{n}\left[-\varepsilon_{t} b_{j k_{t}}[t]\right]_{+} u_{j} & i=k_{t} \\
u_{i} & i \neq k_{t},\end{cases}
\end{aligned}
$$

and

$$
\sigma: \mathbb{R}^{n} \rightarrow \mathbb{R}^{n}, \quad\left(u_{i}\right)_{i=1}^{n} \mapsto\left(u_{i}^{\prime}\right)_{i=1}^{n}, \quad u_{i}^{\prime}=u_{\sigma(i)} .
$$

The sequence (A.18) induces the sequence of the maps

$$
L^{2}\left(\mathbb{R}^{n}\right) \stackrel{\rho_{1}^{*}}{\leftarrow} L^{2}\left(\mathbb{R}^{n}\right) \stackrel{\rho_{2}^{*}}{\leftarrow} \ldots \stackrel{\rho_{m}^{*}}{\leftarrow} L^{2}\left(\mathbb{R}^{n}\right) \stackrel{\sigma^{*}}{\leftarrow} L^{2}\left(\mathbb{R}^{n}\right),
$$

where $\rho_{t}^{*}(f)=f \circ \rho_{t}$ and $\sigma^{*}(f)=f \circ \sigma$ for $f \in L^{2}\left(\mathbb{R}^{n}\right)$.

LEMmA A.8. If the sequence (A.8) is $\sigma$-periodic, the following periodicity holds:

$$
\begin{aligned}
\sigma \circ \rho_{m} \circ \cdots \circ \rho_{1} & =\mathrm{id}, \\
\rho_{1}^{*} \circ \cdots \circ \rho_{m}^{*} \circ \sigma^{*} & =\mathrm{id} .
\end{aligned}
$$

Proof. Suppose that the sequence (A.8) is $\sigma$-periodic. Then, taking $q \rightarrow 1$ limit, the corresponding (classical) $y$-variables in the sequence (4.9) are also $\sigma$-periodic. Then, the associated $c$-vectors in $(4.21)$ are also $\sigma$-periodic. Thus, the associated $g$-vectors [Nak15b], which are not explained here, are also $\sigma$-periodic due to the duality of $c$ - and $g$-vectors [Nak15b, Proposition 3.21]. On the other hand, the linear transformations $\rho_{t}$ and $\sigma$ exactly describe the mutations of $g$-vectors along the sequence (4.9). Therefore, we have the equality (A.22). The equality (A.23) follows from (A.22).

For any invertible linear map $\Upsilon: L^{2}\left(\mathbb{R}^{n}\right) \rightarrow L^{2}\left(\mathbb{R}^{n}\right)$ and any linear operator $\hat{O}$ acting on $L^{2}\left(\mathbb{R}^{n}\right)$, let

$$
\operatorname{Ad}(\Upsilon)(\hat{O}):=\Upsilon \circ \hat{O} \circ \Upsilon^{-1}
$$

Lemma A.9. The following formulas hold: For $t=1, \ldots, m$,

$$
\begin{aligned}
& \operatorname{Ad}\left(\rho_{t}^{*}\right)\left(\hat{u}_{i}\right)= \begin{cases}-\hat{u}_{k_{t}}+d_{k_{t}} \sum_{j=1}^{n}\left[-\varepsilon_{t} b_{j k_{t}}[t]\right]_{+} \hat{u}_{j} & i=k_{t} \\
\hat{u}_{i} & i \neq k_{t},\end{cases} \\
& \operatorname{Ad}\left(\rho_{t}^{*}\right)\left(\hat{p}_{i}\right)= \begin{cases}-\hat{p}_{k_{t}} & i=k_{t} \\
\hat{p}_{i}+d_{k_{t}}\left[-\varepsilon b_{i k_{t}}[t]\right]_{+} \hat{p}_{k_{t}} & i \neq k_{t},\end{cases} \\
& \operatorname{Ad}\left(\sigma^{*}\right)\left(\hat{u}_{i}\right)=\hat{u}_{\sigma(i)}, \quad \operatorname{Ad}\left(\sigma^{*}\right)\left(\hat{p}_{i}\right)=\hat{p}_{\sigma(i)} .
\end{aligned}
$$


Proof. The equalities (A.25) and (A.27) are immediate from the definitions of $\rho_{t}$ and $\sigma$. The equality (A.26) is obtained by applying the chain rule for the inverse of $\rho_{t}$

$$
\begin{aligned}
\left(\rho_{t}\right)^{-1} & : \mathbb{R}^{n} \rightarrow \mathbb{R}^{n}, \quad\left(u_{i}^{\prime}\right)_{i=1}^{n} \mapsto\left(u_{i}\right)_{i=1}^{n}, \\
u_{i} & = \begin{cases}-u_{k_{t}}^{\prime}+d_{k_{t}} \sum_{j=1}^{n}\left[-\varepsilon_{t} b_{j k_{t}}[t]\right]_{+} u_{j}^{\prime} & i=k_{t} \\
u_{i}^{\prime} & i \neq k_{t} .\end{cases}
\end{aligned}
$$

REMARK A.10. In [KN11] the separate symbols $u[t](t=1, \ldots, m+2)$ are employed for the coordinate of each space $\mathbb{R}^{n}$ in the sequence (A.21) from left to right. Here, we do not use them for simplicity.

Let us define the following operators for $t=1, \ldots, m$. (For $t=1$, it is already given in (A.14) and (A.16).)

$$
\begin{aligned}
\hat{w}_{i}[t] & =\sum_{j=1}^{n} b_{j i}[t] \hat{u}_{j}, \quad \hat{D}_{i}[t]=r_{i} \hat{p}_{i}+\hat{w}_{i}[t], \quad \hat{Y}_{i}[t]=\exp \left(\lambda \hat{D}_{i}[t]\right), \\
\hat{Y}^{\alpha}[t] & =\exp (\lambda \alpha \hat{D}[t]), \quad \alpha \hat{D}[t]=\sum_{i=1}^{n} \alpha_{i} \hat{D}_{i}[t] .
\end{aligned}
$$

Then, like the $t=1$ case (A.15), we have the following commutation relations: For $t=1, \ldots, m$,

$$
\begin{aligned}
{\left[\hat{D}_{i}[t], \hat{D}_{j}[t]\right] } & =2 \hbar \sqrt{-1} r_{j} b_{j i}[t], \quad \hat{Y}_{i}[t] \hat{Y}_{j}[t]=q_{j}^{2 b_{j i}[t]} \hat{Y}_{j}[t] \hat{Y}_{i}[t], \\
q^{\langle\alpha, \beta\rangle_{t}} Y^{\alpha}[t] Y^{\beta}[t] & =Y^{\alpha+\beta}[t], \quad\langle\alpha, \beta\rangle_{t}=: \sum_{i, j=1}^{n} \alpha_{i} r_{i} b_{i j}[t] \beta_{j} .
\end{aligned}
$$

Lemma A.11. The following formulas hold: For $t=1, \ldots, m$,

$$
\begin{aligned}
\operatorname{Ad}\left(\rho_{t}^{*}\right)\left(\hat{w}_{i}[t+1]\right) & = \begin{cases}-\hat{w}_{k_{t}}[t] & i=k_{t} \\
\hat{w}_{i}[t]+d_{k_{t}}\left[\varepsilon_{t} b_{k_{t} i}[t]\right]_{+} \hat{w}_{k_{t}}[t] & i \neq k_{t},\end{cases} \\
\operatorname{Ad}\left(\rho_{t}^{*}\right)\left(r_{i} \hat{p}_{i}\right) & = \begin{cases}-r_{k_{t}} \hat{p}_{k_{t}} \\
r_{i} \hat{p}_{i}+d_{k_{t}}\left[\varepsilon_{t} b_{k_{t} i}[t]\right]_{+} r_{k_{t}} \hat{p}_{k_{t}} & i \neq k_{t} .\end{cases}
\end{aligned}
$$

Proof. They follow from Lemma A.9.

REMARK A.12. A subtle difference between the second formulas of (A.26) and (A.34) is important, since $b_{k_{t} i}[t] \neq-b_{i k_{t}}[t]$ in general.

The conclusion of this section is given as follows:

Proposition A.13. (1) Quantum tropical mutations: For $t=1, \ldots, m$, 


$$
\operatorname{Ad}\left(\rho_{t}^{*}\right)\left(\hat{Y}_{i}[t+1]\right)= \begin{cases}\hat{Y}_{k_{t}}[t]^{-1} & i=k_{t} \\ \hat{Y}^{e_{i}+d_{k_{t}}\left[\varepsilon_{t} b_{k_{t}}[t]\right]_{+} e_{k_{t}}[t]} & i \neq k_{t} .\end{cases}
$$

(2) For $t=1, \ldots, m$,

$$
\operatorname{Ad}\left(\rho_{1}^{*}\right) \cdots \operatorname{Ad}\left(\rho_{t}^{*}\right)\left(\hat{Y}_{i}[t+1]\right)=\hat{Y}^{c_{i}[t+1]} .
$$

Proof. (1). This follows from Lemma A.11. (2). Note that the second formula of (4.23) is also written as

$$
c_{i j}[t+1]=c_{i j}[t]+d_{k_{t}}\left(\left[c_{i k_{t}}[t]\right]_{+} b_{k_{t} j}[t]+c_{i k_{t}}[t]\left[-b_{k_{t} j}[t]\right]_{+}\right) \quad j \neq k_{t} .
$$

In particular, for the tropical sign $\varepsilon_{t}$, we have $\left[-\varepsilon_{t} c_{i k_{t}}[t]\right]_{+}=0$. Thus, it is also equivalent to

$$
c_{i j}[t+1]=c_{i j}[t]+d_{k_{t}} c_{i k_{t}}[t]\left[\varepsilon_{t} b_{k_{t} j}[t]\right]_{+} \quad j \neq k_{t} .
$$

Comparing (A.35) and (A.38), we inductively obtain the formula (A.36) for $t=1, \ldots, m$.

\section{A.5. Step 2: Integral expression.}

In the quantum dilogarithm identity (A.10) let us replace the initial quantum $y$ variables $Y_{i}$ with their operator representations $\hat{Y}_{i}$. Then, multiplying the left-hand side of the equality (A.23), we obtain the following equality:

$$
\boldsymbol{\Psi}_{d_{k_{1}}, z_{k_{1}}[1], q_{k_{1}}}\left(\hat{Y}^{\varepsilon_{1} c_{k_{1}}[1]}\right)^{\varepsilon_{1}} \cdots \boldsymbol{\Psi}_{d_{k_{m}}, z_{k_{m}}[m], q_{k_{m}}}\left(\hat{Y}^{\varepsilon_{m} c_{k_{m}}[m]}\right)^{\varepsilon_{m}} \rho_{1}^{*} \cdots \rho_{m}^{*} \sigma^{*}=\mathrm{id}
$$

where the composition symbol $\circ$ is omitted for simplicity.

Using (A.36) repeatedly, it is transformed into the following equality, which is a generalization of the quantum dilogarithm identity in local form in [KN11]:

$$
\begin{aligned}
\boldsymbol{\Psi}_{d_{k_{1}}, z_{k_{1}}[1], q_{k_{1}}}\left(\hat{Y}_{k_{1}}[1]^{\varepsilon_{1}}\right)^{\varepsilon_{1}} \rho_{1}^{*} & \Psi_{d_{k_{2}}, z_{k_{2}}[2], q_{k_{2}}}\left(\hat{Y}_{k_{2}}[2]^{\varepsilon_{2}}\right)^{\varepsilon_{2}} \rho_{2}^{*} \\
& \cdots \boldsymbol{\Psi}_{d_{k_{m}}, z_{k_{m}}[m], q_{k_{m}}}\left(\hat{Y}_{k_{m}}[m]^{\varepsilon_{m}}\right)^{\varepsilon_{m}} \rho_{m}^{*} \sigma^{*}=\mathrm{id} .
\end{aligned}
$$

Using the Dirac's bra-ket notation, we introduce a family of common eigenvectors $|u\rangle$ and $|p\rangle\left(u, p \in \mathbb{R}^{n}\right)$ of the position operators $\hat{u}_{i}$ 's and momentum operators $\hat{p}_{i}$ 's, respectively, and their complex conjugate $\langle u|$ and $\langle p|$. They satisfy

$$
\begin{aligned}
\hat{u}_{i}|u\rangle & =u_{i}|u\rangle, \quad \hat{p}_{i}|p\rangle=p_{i}|p\rangle \\
\left\langle u \mid u^{\prime}\right\rangle & =\prod_{i=1}^{n} \delta\left(u_{i}-u_{i}^{\prime}\right), \quad\left\langle p \mid p^{\prime}\right\rangle=(2 \pi \hbar)^{n} \prod_{i=1}^{n} \delta\left(p_{i}-p_{i}^{\prime}\right), \\
\langle u \mid p\rangle & =\exp \left(\frac{\sqrt{-1}}{\hbar} u p\right), \quad\langle p \mid u\rangle=\exp \left(-\frac{\sqrt{-1}}{\hbar} u p\right), \quad u p=\sum_{i=1}^{n} u_{i} p_{i} .
\end{aligned}
$$

In particular, we have, for vectors $\langle u|$ and $|p\rangle$, 


$$
\frac{\left\langle u\left|\hat{D}_{i}[t]\right| p\right\rangle}{\langle u \mid p\rangle}=r_{i} p_{i}+w_{i}[t], \quad w_{i}[t]=\sum_{j=1}^{n} b_{j i}[t] u_{j}
$$

We also have the completeness property,

$$
1=\int d u|u\rangle\left\langle u\left|, \quad 1=\int \frac{d p}{(2 \pi \hbar)^{n}}\right| p\right\rangle\langle p| .
$$

Let

$$
\hat{O}=\Psi_{d_{k_{1}}, z_{k_{1}}[1], q_{k_{1}}}\left(\hat{Y}_{k_{1}}[1]\right)^{\varepsilon_{1}} \rho_{1}^{*} \cdots \boldsymbol{\Psi}_{d_{k_{m}}, z_{k_{m}}[m], q_{k_{m}}}\left(\hat{Y}_{k_{m}}[m]\right)^{\varepsilon_{m}} \rho_{m}^{*} \sigma^{*}
$$

be the left-hand side of (A.40), which is actually the identity operator due to (A.40). Choose an arbitrary position eigenvector $|u[1]\rangle$. Then, we introduce the momentum eigenvector $|\tilde{p}[1]\rangle$ whose eigenvalues are given by

$$
\tilde{p}_{i}[1]=w_{i}[1]:=\sum_{i=1}^{n} b_{j i}[1] u_{j}[1] .
$$

Let

$$
F(u[1], \tilde{p}[1]):=\frac{\langle u[1]|\hat{O}| \tilde{p}[1]\rangle}{\langle u[1] \mid \tilde{p}[1]\rangle},
$$

which is actually 1. Skipping some detail (see [KN11, Sections 5.2 and A.3]), we obtain the following integral expression, using (A.44):

$$
\begin{aligned}
F(u[1], \tilde{p}[1])= & (2 \pi \hbar)^{-n(m-1)} \int d p[1] \cdots d p[m-1] d u[2] \ldots d u[m] \\
& \times \boldsymbol{\Psi}_{d_{k_{1}}, z_{k_{1}}[1], q_{k_{1}}}\left(y_{k_{1}}[1]^{\varepsilon_{1}}\right)^{\varepsilon_{1}} \exp \left(\frac{\sqrt{-1}}{\hbar} u[1](p[1]-\tilde{p}[1])\right) \cdots \\
& \times \boldsymbol{\Psi}_{d_{k_{m}}, z_{k_{m}}[m], q_{k_{m}}}\left(y_{k_{m}}[m]^{\varepsilon_{m}}\right)^{\varepsilon_{m}} \exp \left(\frac{\sqrt{-1}}{\hbar} u[m](p[m]-\tilde{p}[m])\right),
\end{aligned}
$$

where $p_{i}[m]$ is determined by

$$
r_{\sigma^{-1}(i)} \tilde{p}_{\sigma^{-1}(i)}[1]= \begin{cases}-r_{k_{m}} p_{k_{m}}[m] & i=k_{m} \\ r_{i} p_{i}[m]+d_{k_{m}}\left[\varepsilon_{m} b_{k_{m} i}[m]\right]_{+} r_{k_{m}} p_{k_{m}}[m] & i \neq k_{m}\end{cases}
$$

while $\tilde{p}_{i}[t](t=2, \ldots, m)$ and $y_{k_{t}}[t](t=1, \ldots, m)$ are dependent variables of the integration variables such that, for $t=1, \ldots, m-1$,

$$
r_{i} \tilde{p}_{i}[t+1]= \begin{cases}-r_{k_{t}} p_{k_{t}}[t] & i=k_{t} \\ r_{i} p_{i}[t]+d_{k_{t}}\left[\varepsilon_{t} b_{k_{t} i}[t]\right]_{+} r_{k_{t}} p_{k_{t}}[t] & i \neq k_{t},\end{cases}
$$

and, for $t=1, \ldots, m$, 


$$
y_{k_{t}}[t]:=\exp \left(\lambda\left(r_{k_{t}} p_{k_{t}}[t]+w_{k_{t}}[t]\right)\right), \quad w_{k_{t}}[t]=\sum_{i=1}^{n} b_{j k_{t}}[t] u_{j}[t] .
$$

REMARK A.14. The symmetry of the skew-symmetrizer (4.11) is used to obtain the relation (A.50). Without it, the left-hand side of (A.50) is $r_{i} \tilde{p}_{\sigma^{-1}(i)}$, which we do not want. (Compare with the forthcoming (A.64).)

\section{A.6. Step 3: Saddle point method.}

In our parametrization of $q$ in (A.11), the asymptotic behavior of the quantum dilogarithms of higher degree in (A.2) is expressed as

$$
\boldsymbol{\Psi}_{d, z, q_{i}}(x) \sim \exp \left(\frac{\sqrt{-1}}{\hbar} \frac{1}{2 \lambda^{2} r_{i}} \operatorname{Li}_{2 ; d, z}(-x)\right), \quad \hbar \rightarrow 0^{+} .
$$

We are interested in the asymptotic behavior of (A.49) in the limit $\hbar \rightarrow 0^{+}$. Thus, we may replace the quantum dilogarithms therein by the right-hand side of (A.53), and we have

$$
\begin{aligned}
F(u[1], \tilde{p}[1]) \sim & (2 \pi \hbar)^{-n(m-1)} \int d p[1] \cdots d p[m-1] d u[2] \cdots d u[m] \\
& \times \exp \left(\frac{\sqrt{-1}}{\hbar} \sum_{t=1}^{m}\left\{\frac{\varepsilon_{t}}{2 \lambda^{2} r_{k_{t}}} \operatorname{Li}_{2 ; d_{k_{t}}, z_{k_{t}}[t]}\left(-y_{k_{t}}[t]^{\varepsilon_{t}}\right)+u[t](p[t]-\tilde{p}[t])\right\}\right) .
\end{aligned}
$$

REmark A.15. Due to our assumption of $\lambda \approx 1$ with $\operatorname{Im} \lambda>0, y_{k_{t}}[t]$ defined by (A.52) is a complex number close to a positive real number. Accordingly, the functions $\mathrm{Li}_{2 ; d_{k_{t}}, z_{k_{t}}[t]}(x)$ in (A.54) are analytically continued in the vicinity of the negative real line $\mathbb{R}_{-}$.

To evaluate the integral (A.54) in the limit $\hbar \rightarrow 0^{+}$, we apply the saddle point method.

To do that, we need the following formulas.

Lemma A.16.

$$
\begin{aligned}
r_{i} \frac{\partial}{\partial u_{i}[t]}\left(\frac{\varepsilon_{t}}{2 \lambda^{2} r_{k_{t}}} \operatorname{Li}_{2 ; d_{k_{t}}, z_{k_{t}}[t]}\left(-y_{k_{t}}[t]^{\varepsilon_{t}}\right)\right) & =-\frac{1}{\lambda} \log \left(P_{d_{k_{t}}, z_{k_{t}}[t]}\left(y_{k_{t}}[t]^{\varepsilon t}\right)\right)^{-b_{k_{t}}[t] / 2}, \\
\frac{\partial}{\partial p_{i}[t]}\left(\frac{\varepsilon_{t}}{2 \lambda^{2} r_{k_{t}}} \operatorname{Li}_{2 ; d_{k_{t}}, z_{k_{t}}[t]}\left(-y_{k_{t}}[t]^{\varepsilon_{t}}\right)\right) & =\delta_{i k_{t}} \frac{1}{\lambda} \log \left(P_{d_{k_{t}}, z_{k_{t}}[t]}\left(y_{k_{t}}[t]^{\varepsilon_{t}}\right)\right)^{-1 / 2} .
\end{aligned}
$$

Proof. These are obtained by (3.3), (A.52), and the skew-symmetric property of $R B[t]$.

The saddle point equation of the integral (A.54) is the extremum condition of its integrand with respect to the integral variables $p[t](t=1, \ldots, m-1)$ and $u[t](t=$ $2, \ldots, m)$. 
(a). Extremum condition for $u_{i}(t)(t=2, \ldots, m)$. By differentiating the integrand of (A.54) by $u_{i}(t)$ using (A.55), and multiplying $r_{i}$, we have

$$
-\frac{1}{\lambda} \log \left(P_{d_{k_{t}}, z_{k_{t}}[t]}\left(y_{k_{t}}[t]^{\varepsilon_{t}}\right)\right)^{-b_{k_{t} i}[t] / 2}+r_{i} p_{i}[t]-r_{i} \tilde{p}_{i}[t]=0 .
$$

Note that, in particular,

$$
p_{k_{t}}[t]=\tilde{p}_{k_{t}}[t]
$$

By (A.57) and (A.51), we also have, for $t=2, \ldots, m-1$,

$$
e^{\lambda r_{i} \tilde{p}_{i}[t+1]}= \begin{cases}\left(e^{\lambda r_{k_{t}} \tilde{p}_{k_{t}}[t]}\right)^{-1} & i=k_{t} \\ e^{\lambda r_{i} \tilde{p}_{i}[t]}\left(\left(e^{\lambda r_{k_{t}} \tilde{p}_{k_{t}}[t]}\right)^{\left[\varepsilon_{t} b_{k_{t}}[t]\right]_{+}}\right)^{d_{k_{t}}} & \\ \quad \times\left(P_{d_{k_{t}}, z_{k_{t}}[t]}\left(y_{k_{t}}[t]^{\varepsilon_{t}}\right)\right)^{-b_{k_{t} i}[t] / 2} & i \neq k_{t} .\end{cases}
$$

(b). Extremum condition for $p_{i}(t)(t=1, \ldots, m-1)$. By differentiating the integrand of (A.54) by $p_{i}(t)$ using (A.56) and (A.51), we have, for $i=k_{t}$,

$$
\begin{aligned}
\frac{1}{\lambda} \log & \left(P_{d_{k_{t}}, z_{k_{t}}[t]}\left(y_{k_{t}}[t]^{\varepsilon_{t}}\right)\right)^{-1 / 2}+u_{k_{t}}[t] \\
& -\sum_{j=1}^{n} d_{k_{t}}\left[\varepsilon_{t} b_{k_{t}}[t]\right]_{+} u_{j}[t+1]+u_{k_{t}}[t+1]=0,
\end{aligned}
$$

and otherwise,

$$
u_{i}[t]-u_{i}[t+1]=0, \quad i \neq t_{k}
$$

By (A.60) and (4.2) ( noticing that $\left[-b_{i k}\right]_{+} b_{k j}+b_{i k}\left[b_{k j}\right]_{+}=\left[b_{i k}\right]_{+} b_{k j}+b_{i k}\left[-b_{k j}\right]_{+}$), we also have the following equations for $w_{i}[t]=\sum_{j=1}^{n} b_{j i}[t] u_{j}[t]$ for $t=1, \ldots, m-1$ :

$$
e^{\lambda w_{i}[t+1]}= \begin{cases}\left(e^{\lambda w_{k_{t}}[t]}\right)^{-1} & i=k_{t} \\ e^{\lambda w_{i}[t]}\left(\left(e^{\lambda w_{k_{t}}[t]}\right)^{\left.\left[\varepsilon_{t} b_{k_{t}}[t]\right]\right]_{+}}\right)^{d_{k_{t}}} & \\ \left.\times\left(P_{d_{k_{t}}, z_{k_{t}}[t]}\left(y_{k_{t}}[t]\right]^{\varepsilon_{t}}\right)\right)^{-b_{k_{t} i}[t] / 2} & i \neq k_{t} .\end{cases}
$$

A complex (but almost positive real) solution $u_{i}[t](t=2, \ldots, m), p_{i}[t](t=$ $1, \ldots, m-1)$ of $(\mathrm{A} .57)$ and (A.60) is constructed as follows.

(1). ( $y$-variables) We have $u_{i}[1]$ as initial data, from which $w_{i}[1]$ is uniquely determined. We set $y_{i}[1]=\exp \left(2 \lambda w_{i}[1]\right)$. Then, $y_{i}[t](t=1, \ldots, m)$ are determined by the mutation sequence (4.9).

(2). ( $u$-variables) We determine $u_{i}[t](t=2, \ldots, m)$ by the extremum condition (A.60) and (A.61).

(3). ( $p$-variables) Set $\tilde{p}_{i}[t](t=2, \ldots, m)$ by $\exp \left(\lambda r_{i} \tilde{p}_{i}[t]\right)=y_{i}[t]^{1 / 2}$. Then, determine $p_{i}[t](t=1, \ldots, m-1)$ by (A.51), and $p_{i}[m]$ by (A.50). 
It is necessary to check that (A.52) and (A.57) are satisfied.

The condition (A.52): By (A.60) and (A.61), we have (A.62). Thus, we have $\exp \left(\lambda r_{i} \tilde{p}_{i}[t]\right)=\exp \left(\lambda w_{i}[t]\right)=y_{i}[t]^{1 / 2}$. Therefore, the condition (A.52) is satisfied.

The condition (A.57): Since $\exp \left(\lambda r_{i} \tilde{p}_{i}[t]\right)=y_{i}[t]^{1 / 2}$, the condition (A.59) is satisfied. Then, combining it with (A.51), we obtain (A.57) for $t=1, \ldots, m-1$. To obtain (A.57) for $t=m$ requires a little extra consideration. Extend the above construction for $y_{i}[m+1]$ and $\tilde{p}_{i}[m+1]$. Then, the condition (A.59) is satisfied for $t=m$. Moreover, thanks to the $\sigma$-periodicity of $y$-variables, we have

$$
r_{\sigma(i)} \tilde{p}_{\sigma(i)}[m+1]=r_{i} \tilde{p}_{i}[1] .
$$

Thus, we have

$$
e^{\lambda r_{\sigma^{-1}(i)} \tilde{p}_{\sigma^{-1}(i)}[1]}= \begin{cases}\left(e^{\lambda r_{k_{m}} \tilde{p}_{k_{m}}[m]}\right)^{-1} & i=k_{m} \\ e^{\lambda r_{i} \tilde{p}_{i}[m]}\left(\left(e^{\lambda r_{k_{m}} \tilde{p}_{k_{m}}[m]}\right)^{\left[\varepsilon_{t} b_{k_{m} i}[m]\right]_{+}}\right)^{d_{k_{m}}} & \\ \quad \times\left(P_{d_{k_{m}}, z_{k_{m}}[m]}\left(y_{k_{m}}[m]^{\varepsilon_{m}}\right)\right)^{-b_{k_{m}}[m] / 2} & i \neq k_{m} .\end{cases}
$$

Then, combining it with (A.50), we obtain (A.57) for $t=m$.

Thus, this is indeed a solution of the saddle point equation.

The saddle point method claims that, under "some" condition which we do not pursue in this paper, the integral (A.54) in the limit $\hbar \rightarrow 0$ is approximated at the value of the integrand at an extremum point, up to some multiplicative factor which is irrelevant here. (See [Tak08, p. 95] for the explicit expression for the one variable case.) Therefore, taking the above solution, ignoring the multiplicative factor, then taking the logarithm and removing the factor $\sqrt{-1} / \hbar$, we have

$$
\sum_{t=1}^{m}\left\{\frac{\varepsilon_{t}}{2 \lambda^{2} r_{k_{t}}} \operatorname{Li}_{2 ; d_{k_{t}}, z_{k_{t}}[t]}\left(-y_{k_{t}}[t]^{\varepsilon_{t}}\right)+u[t](p[t]-\tilde{p}[t])\right\} .
$$

Recall that, for our solution,

$$
\begin{aligned}
p_{i}[t]-\tilde{p}_{i}[t] & =\frac{1}{\lambda r_{i}} \log \left(P_{d_{k_{t}}, z_{k_{t}}[t]}\left(y_{k_{t}}[t]^{\varepsilon_{t}}\right)\right)^{-b_{k_{t}}[t] / 2}, \\
w_{i}[t] & =\frac{1}{2 \lambda} \log y_{i}[t] .
\end{aligned}
$$

Thus, using the skew-symmetric property $b_{k_{t} i}[t] r_{i}^{-1}=-b_{i k_{t}}[t] r_{k_{t}}^{-1}$, we have

$$
\begin{aligned}
\sum_{i=1}^{n} u_{i}[t]\left(p_{i}[t]-\tilde{p}_{i}[t]\right) & =\frac{1}{\lambda} \sum_{i=1}^{n} \frac{1}{r_{i}}\left(-\frac{b_{k_{t} i}[t]}{2}\right) u_{i}[t] \log P_{d_{k_{t}}, z_{k_{t}}[t]}\left(y_{k_{t}}[t]^{\varepsilon_{t}}\right) \\
& =\frac{1}{2 \lambda r_{k_{t}}} \sum_{i=1}^{n} b_{i k_{t}}[t] u_{i}[t] \log P_{d_{k_{t}}, z_{k_{t}}[t]}\left(y_{k_{t}}[t]^{\varepsilon_{t}}\right) \\
& =\frac{1}{2 \lambda r_{k_{t}}} w_{k_{t}}[t] \log P_{d_{k_{t}}, z_{k_{t}}[t]}\left(y_{k_{t}}[t]^{\varepsilon_{t}}\right) \\
& =\frac{\varepsilon_{t}}{4 \lambda^{2} r_{k_{t}}} \log y_{i}[t]^{\varepsilon_{t}} \cdot \log P_{d_{k_{t}}, z_{k_{t}}[t]}\left(y_{k_{t}}[t]^{\varepsilon_{t}}\right)
\end{aligned}
$$


Thus, the expression (A.65) is equal to

$$
\frac{-1}{2 \lambda^{2}} \sum_{t=1}^{m} \frac{\varepsilon_{t}}{r_{t_{k}}}\left\{-\operatorname{Li}_{2 ; d_{k_{t}}, z_{k_{t}}[t]}\left(-y_{k_{t}}[t]^{\varepsilon_{t}}\right)-\frac{1}{2} \log y_{i}[t]^{\varepsilon_{t}} \cdot \log P_{d_{k_{t}}, z_{k_{t}}[t]}\left(y_{k_{t}}[t]^{\varepsilon_{t}}\right)\right\},
$$

which exactly yields the Rogers dilogarithms of higher degree (3.5). On the other hand, this term is 0 from the beginning. Therefore, we have the classical dilogarithm identity of higher degree (4.28) with complex (almost real) argument. Taking $\lambda \rightarrow 1$, we recover the identity (4.28) with real argument.

\section{References}

[Blo78] S. Bloch, Applications of the dilogarithm function in algebraic $K$-theory and algebraic geometry, Proceedings of the International Symposium on Algebraic Geometry, Kinokuniya Book Store, Tokyo, 1978, 103-114.

[Cha05] F. Chapoton, Functional identities for the Rogers dilogarithm associated to cluster Ysystems, Bull. London Math. Soc., 37 (2005), 755-760.

[CS14] L. Chekhov and M. Shapiro, Teichmüller spaces of Riemann surfaces with orbifold points of arbitrary order and cluster variables, Int. Math. Res. Notices, 2014 (2014), 2746-2772.

[FC99] V. V. Fock and L. Chekhov, Quantum Teichmüller space, Theor. Math. Phys., 120 (1999), 1245-1259.

[FG09a] V. V. Fock and A. B. Goncharov, Cluster ensembles, quantization and the dilogarithm, Annales Sci. de l'École Norm. Sup., 42 (2009), 865-930.

[FG09b] V. V. Fock and A. B. Goncharov, The quantum dilogarithm and representations of quantum cluster varieties, Invent. Math., 175 (2009), 223-286.

[FK94] L. D. Faddeev and R. M. Kashaev, Quantum dilogarithm, Mod. Phys. Lett., A9 (1994), 427-434.

[FS95] E. Frenkel and A. Szenes, Thermodynamic Bethe ansatz and dilogarithm identities, I, Math. Res. Lett., 2 (1995), 677-693.

[FV93] L. D. Faddeev and A. Yu. Volkov, Abelian current algebra and the Virasoro algebra on the lattice, Phys. Lett., 315 (1993), 311-318.

[FZ02] S. Fomin and A. Zelevinsky, Cluster algebras I: Foundations, J. Amer. Math. Soc., 15 (2002), 497-529.

[FZ07] S. Fomin and A. Zelevinsky, Cluster algebras IV: Coefficients, Compositio Math., 143 (2007), 112-164.

[GHKK18] M. Gross, P. Hacking, S. Keel and M. Kontsevich, Canonical bases for cluster algebras, J. Amer. Math. Soc., 31 (2018), 497-608.

$\left[\mathrm{IIK}^{+} 13\right] \quad$ R. Inoue, O. Iyama, B. Keller, A. Kuniba and T. Nakanishi, Periodicities of T and Ysystems, dilogarithm identities, and cluster algebras I: Type $B_{r}$, Publ. RIMS, 49 (2013), $1-42$.

[Kel11] B. Keller, On cluster theory and quantum dilogarithm identities, Representations of algebras and related topics (eds. A. Skowroński and K. Yamagata), EMS Series of Congress Reports, European Math. Soc., 2011, 85-116.

[Kir95] A. N. Kirillov, Dilogarithm identities, Prog. Theor. Phys. Suppl., 118 (1995), 61-142.

[KN11] R. M. Kashaev and T. Nakanishi, Classical and quantum dilogarithm identities, SIGMA, 7 (2011), 102, 29 pages.

[KS08] M. Kontsevich and Y. Soibelman, Stability structures, Donaldson-Thomas invariants and cluster transformations, 2008, arXiv:0811.2435 [math.AG].

[Lew81] L. Lewin, Polylogarithms and associated functions, North-Holland, Amsterdam, 1981.

[Nag11] K. Nagao, Wall-crossing of the motivic Donaldson-Thomas invariants, 2011, arXiv: 1103.2922 [math.AG].

[Nak11a] T. Nakanishi, Dilogarithm identities for conformal field theories and cluster algebras: Sim- 
ply laced case, Nagoya Math. J., 202 (2011), 23-43.

[Nak11b] T. Nakanishi, Periodicities in cluster algebras and dilogarithm identities, Representations of algebras and related topics (eds. A. Skowroński and K. Yamagata), EMS Series of Congress Reports, European Math. Soc., 2011, 407-444.

[Nak15a] T. Nakanishi, Quantum generalized cluster algebras and quantum dilogarithms of higher degrees, Theor. Math. Phys., 185 (2015), 1759-1768.

[Nak15b] T. Nakanishi, Structure of seeds in generalized cluster algebras, Pacific J. Math., 277 (2015), 201-218.

[NR16] T. Nakanishi and D. Rupel, Companion cluster algebras to a generalized cluster algebra, Travaux Mathématiques, 24 (2016), 129-149.

[Rup13] D. Rupel, Greedy bases in rank 2 generalized cluster algebras, 2013, arXiv:1309.2567 [math.RA].

[Tak08] L. A. Takhtajan, Quantum mechanics for mathematicians, American Mathematical Society, Providence, RI, 2008.

[Zag07] D. Zagier, The dilogarithm function, Frontiers in Number Theory, Physics, and Geometry II, Springer, Berlin, Heidelberg, 2007, 3-65.

\section{Tomoki NAKANISHI}

Graduate School of Mathematics

Nagoya University

Chikusa-ku

Nagoya 464-8602, Japan

E-mail: nakanisi@math.nagoya-u.ac.jp 